\title{
Correction of vasopressin deficit in the lateral septum ameliorates social deficits of mouse autism model
}

\author{
Amélie M. Borie,, ${ }^{1}$ Yann Dromard, ${ }^{1}$ Gilles Guillon, ${ }^{1}$ Aleksandra Olma, ${ }^{2}$ Maurice Manning, ${ }^{2}$ Françoise Muscatelli, ${ }^{3}$ \\ Michel G. Desarménien, ${ }^{1}$ and Freddy Jeanneteau ${ }^{1}$ \\ 'Montpellier University, CNRS, INSERM, Institut de Génomique Fonctionnelle, Montpellier, France. ${ }^{2}$ College of Medicine and Life Sciences, University of Toledo, Toledo, Ohio, USA. Institut des Neurosciences \\ de la Méditerranée, INSERM, Aix-Marseille University, Marseille, France.
}

\begin{abstract}
Intellectual and social disabilities are common comorbidities in adolescents and adults with MACE family member L2 (MAGEL2) gene deficiency characterizing the Prader-Willi and Schaaf-Yang neurodevelopmental syndromes. The cellular and molecular mechanisms underlying the risk for autism in these syndromes are not understood. We asked whether vasopressin functions are altered by MAGEL2 deficiency and whether a treatment with vasopressin could alleviate the disabilities of social behavior. We used Magel2-knockout mice (adult males) combined with optogenetic or pharmacological tools to characterize disease modifications in the vasopressinergic brain system and monitor its impact on neurophysiological and behavioral functions. We found that the activation of vasopressin neurons and projections in the lateral septum were inappropriate for performing a social habituation/discrimination task. Mechanistically, the lack of vasopressin impeded the deactivation of somatostatin neurons in the lateral septum, which predicted social discrimination deficits. Correction of vasopressin septal content by administration or optogenetic stimulation of projecting axons suppressed the activity of somatostatin neurons and ameliorated social behavior. This preclinical study identified vasopressin in the lateral septum as a key factor in the pathophysiology of Magel2-related neurodevelopmental syndromes.
\end{abstract}

\section{Introduction}

Autism spectrum disorders (ASDs) affect 1 in 68 people and are characterized by difficulties with communication, restrictive interests, and repetitive behaviors influencing the ability to function properly (1). Treatment options remain limited and pharmacological alternatives such as risperidone and aripiprazole come with side effects (2).

A tremendous genetic heterogeneity between patients presents challenges for developing treatments. The majority of ASDs cannot be ascribed to a single genetic or environmental cause and therefore most likely arise from a complex interplay of genetics and environment (3). Clustering of genes associated with a risk of ASD prevalence highlighted pathophysiological pathways, including synaptic, epigenetic, and translational regulation (4). Considering a Research Domain Criteria project approach to ASD may strongly clarify mechanisms and open the way to new therapies extended to more common psychiatric symptoms like social deficits (5).

Neurodevelopmental disorders characterized by mutations of chromosome 15q11-13 exhibit penetrant risk for ASDs, indicating the importance of genes in this locus for the pathophysiology (6, 7). It is a large chromosomal deletion of 15q11-13 in Prader-Willi

Authorship note: MCD and FJ contributed equally to this work.

Conflict of interest: The authors have declared that no conflict of interest exists.

Copyright: () 2021, American Society for Clinical Investigation.

Submitted: September 21, 2020; Accepted: November 18, 2020;

Published: January 19, 2021.

Reference information: J Clin Invest. 2021;131(2):e144450.

https://doi.org/10.1172/JCl144450.
Syndrome (PWS) and a disruption of one gene in this locus, MAGE family member L2 (MAGEL2), in Schaaf-Yang Syndrome (SYS) that provide $25 \%$ and $75 \%$ risk for ASD, respectively $(6,7) . M A G E L 2$ is a maternally imprinted, paternally expressed gene central to the pathophysiology of PWS and SYS (8). Its deficiency interferes with developmental functions essential for setting multiscale organization of the nervous system controlling muscle tone and feeding (9, 10). Common symptomatic features of MAGEL2-related diseases are hypotonia, feeding difficulties during early life, social disabilities, and intellectual and/or developmental delay $(11,12)$.

In a mouse model of SYS and PWS, which demonstrated construct and face validity, mice lacking the Magel2 gene presented hypotonia, feeding difficulties during early life, and social deficits. Treatment with oxytocin (OXT) around birth restores feeding in Magel2-KO mice (15) and in PWS patients (16), suggesting predictive validity of this mouse model.

Adult Magel2-KO mice (male only) also present ASD-like features ameliorated by OXT treatment around birth $(14,15)$. Longterm benefits of OXT treatment are difficult to predict and restricted to a therapeutic period around birth $(<5$ months based on trials NCTO2205034 and NCT04283578 registered at ClinicalTrials. gov) that is not accessible for most ASD cases diagnosed later in life. In adult ASD patients, OXT showed no long-term benefits 1 year after administration (17). In adult mouse models of ASD, OXT showed acute impact on social deficits (18-20). Therefore, it is essential to characterize other treatments that would benefit patients diagnosed after the perinatal therapeutic period.

Vasopressin (AVP), a peptide sharing many features with OXT (21), is also important for the regulation of social behavior and 
a focus of recent clinical studies. Patients with ASD improved social communication upon treatment with AVP (22) as well as with antagonists of AVP receptor subtype 1A (AVPR1A) (23). The positive outcomes of both trials demand clarity about the mechanisms underlying AVPR responses in ASD and related diseases like PWS and SYS.

We hypothesized that the AVP system would be impaired in Magel2-KO mice, but the efficacy of AVP treatment has never been tested to our knowledge. Here, we devised strategies to understand the effect of Magel 2 mutation on the AVP system and modify the pathophysiology.

\section{Results}

EEG monitoring during social and nonsocial exploratory behavior. The effects of Magel 2 mutation were assessed on brain activity (theta oscillations 4-12 Hz) in adult males because brain activity can be modulated by social stimuli (24). We used a telemetric system to record the EEG from wire electrodes chronically implanted atop the somatosensory cortex. Animals were subjected to 4 successive encounters with an unfamiliar juvenile followed by another encounter with a different juvenile to assess discrimination (Figure 1A). The analysis of EEG power spectral density in the theta band $(4-12 \mathrm{~Hz})$ showed distinct modulation between controls $\left(\right.$ Magel2 $\left.^{+/+}\right)$and mutants (Magel2 $\left.{ }^{+/-p}\right)$ at the time of social novelty and discrimination (Figure 1B). Particularly, the EEG power decreased in the $2-8 \mathrm{~Hz}$ band, whereas it increased in the $9-15 \mathrm{~Hz}$ band of $\mathrm{Magel2}^{+/+}$mice, an effect absent in Magel2 ${ }^{+/ \mathrm{p}}$ mice (Supplemental Figure 1; supplemental material available online with this article; https://doi.org/10.1172/JCI144450DS1). Neurophysiological defects corresponded with the alteration of behavioral exploration at the time of social novelty and discrimination in Magel2 ${ }^{+/-p}$ mice compared with Magel2 ${ }^{+/+}$controls (Figure 1C). The effects of Magel2 mutation were specific to social stimuli given that no difference from controls was observed during encounters with familiar and unfamiliar objects (Figure 1, D and E).

Results of c-Fos mapping of septal neurons activated by social exploration. Social discrimination in rodents depends on a neurocircuit between the hippocampus and lateral septum (LS) modulated by OXT (25) and AVP (26). This suggests a possible role for the LS and its modulation by neuropeptides in Magel2-related pathophysiology. The effects of Magel2 mutation were assessed on LS activation using c-Fos mapping. First, c-Fos induction at social novelty was more robust than after exploration of a novel object in $\mathrm{Magel2}^{+/+}$mice (Figure 2A). Second, c-Fos expression was higher at baseline in LS subregions of Magel2 $^{+/-p}$ mice than Magel2 ${ }^{+/+}$controls (2-way ANOVA $F_{1,33}=9.65, P=0.0039$, Figure 2B). Third, c-Fos induction was less reactive to social novelty and discrimination in the LS of Magel2 $^{+/-p}$ mice than Magel2 $^{+/+}$controls (Figure 2C). In particular, the somatostatin (SST) neurons, which control a variety of social behaviors (27-29), induced c-Fos in the dorsal LS at social novelty, and levels returned to baseline before social discrimination (Figure 2D). Analysis of an additional time point, 2 hours after social novelty (without subsequent encounters), showed that c-Fos induction in SST neurons was persistent in Magel2 ${ }^{+/-p}$ mice but not in controls (Figure 2D). Yet, the number of SST cells $/ \mathrm{mm}^{2}$ in the dorsal LS did not differ between groups $\left(31\right.$ Magel2 $^{+/+} 235 \pm 20$ and 30 Magel2 $^{+/-p} 226 \pm 22$, unpaired $t$ test
$P=0.77)$. Finally, injection of AVP $(30 \mu \mathrm{M})$ directly in the LS via bilateral cannulas immediately after social novelty reduced the expression of c-Fos in SST neurons of the dorsal LS compared with vehicle-injected controls (Figure 2E).

Expression of AVP receptors in SST neurons of the dorsal LS. The effects of Magel2 mutation were assessed on the expression of AVPRs in the LS. Binding studies with the radioligand [ $\left.{ }^{125} \mathrm{I}\right] \mathrm{LVA}$ $(1 \mathrm{nM})$ on frozen brain sections revealed no difference between genotypes (Figure 3A). To gain cellular precision, we synthesized a fluorescent peptide based on the selective AVPR ligand d[Lys $\left.{ }^{8}\right]$ VP (30). Conjugation of Alexa Fluor 647 to d[Lys $\left.{ }^{8}\right]$ VP provided a new ligand d[Lys(Alexa Fluor 647) ${ }^{8}$ ]VP exhibiting the following binding affinities on mouse receptors (in $\mathrm{nM}$ ): $3.7 \pm 0.2$ for OXT receptor (OXTR), $765 \pm 182$ for AVPR1A, $200 \pm 36$ for AVPR1B, and 10,429 \pm 745 for AVPR2. This fluorescent peptide, which does not bind to cells fixed with paraformaldehyde, was injected through cannulas directly in the LS $(50 \mu \mathrm{M})$ with and without the OXTR competitor (TGOT $5 \mu \mathrm{M}$ ) or the AVPR/OXTR competitor (Manning compound $100 \mu \mathrm{M}$ ). The condition selected to detect AVPR binding sites revealed 3 types of AVPR-expressing cells in the LS: the bright, dim, and blood vessels (Figure 3B). Specifically, AVPR binding sites were detected in neurons marked with GAD67 (dim cells), among which half colabeled with SST antibodies. The bright cells colabeled with neurogranin (principal neurons) (Figure 3C). Magel2 mutation reduced the proportion of SST cells with AVPR binding sites (Figure 3D), although it did not alter the overall number of cells labeled with d[Lys(Alexa Fluor 647) ${ }^{8}$ ]VP in the LS.

Electrophysiological monitoring of septal neuron response to AVP. The effects of Magel2 mutation were assessed on neuronal activity in the dorsal LS at baseline and in response to bath application of AVP $(1 \mu \mathrm{M})$. We used patch clamp recordings in acute coronal brain slices to characterize responses based on changes of firing rate with AVP. A majority of cells randomly patched in the dorsal LS had an increased firing rate in response to AVP application, whereas the rest were insensitive to or inhibited by AVP (Figure $4 \mathrm{~A})$. The effect of AVP took minutes to develop and tens of minutes to washout, a typical response unaffected by Magel 2 mutation (Figure 4, B and C). Nevertheless, Magel2 deficiency altered the proportion of typical responses. More cells in the dorsal LS were insensitive to AVP in Magel2 ${ }^{+/-p}$ mice than in Magel2 ${ }^{+/+}$controls. These observations made in whole cell recordings were confirmed with additional experiments performed in a loose patch configuration (Figure 4D). The injection of a fluorescent dye in the pipette permitted the identification of the very scarce inhibited cells in Magel2 ${ }^{+/-p}$ mice with SST antibodies (Figure 4E).

The inputs on the excited and inhibited cells were also characterized by monitoring synaptic events at baseline and in response to AVP (Figure 4F and Table 1). The major effect of AVP in the dorsal LS was to increase the frequency of inhibitory synaptic events on both cell types, which was blocked by a GABA receptor blocker $(0.3$ $\mu \mathrm{M})$ and an $\mathrm{Na}^{+}$channel blocker $(0.3 \mu \mathrm{M})$ (Table 1$)$. Remarkably, AVP had no effect on the frequency of excitatory synaptic events.

Effect of intraseptal AVP on behavior and neurophysiology. To promote AVP-mediated neuromodulation in the dorsal LS of Magel2 ${ }^{+/-p}$ mice, we cannulated the dorsal LS bilaterally to inject AVP $(30 \mu \mathrm{M})$ or the Manning compound at a dose that blocked 
A
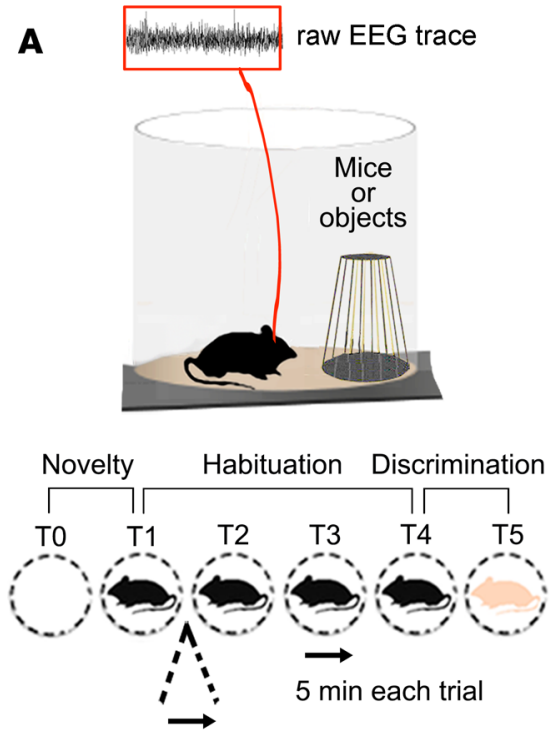

20 min

inter trials

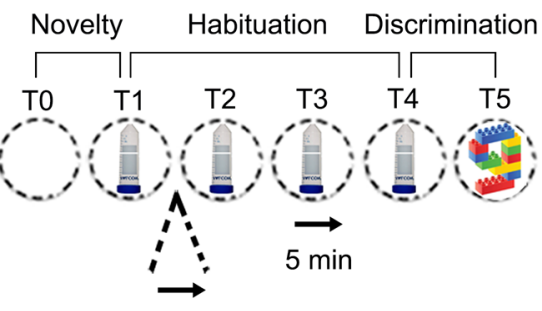

$20 \mathrm{~min}$
B

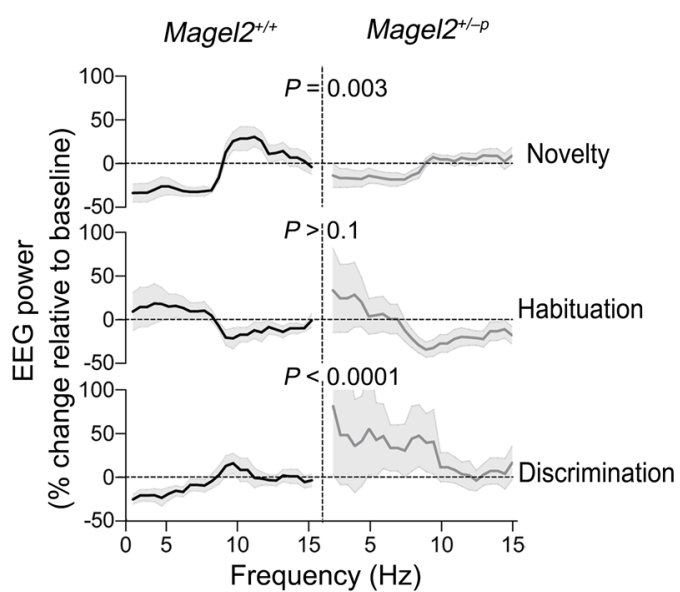

D

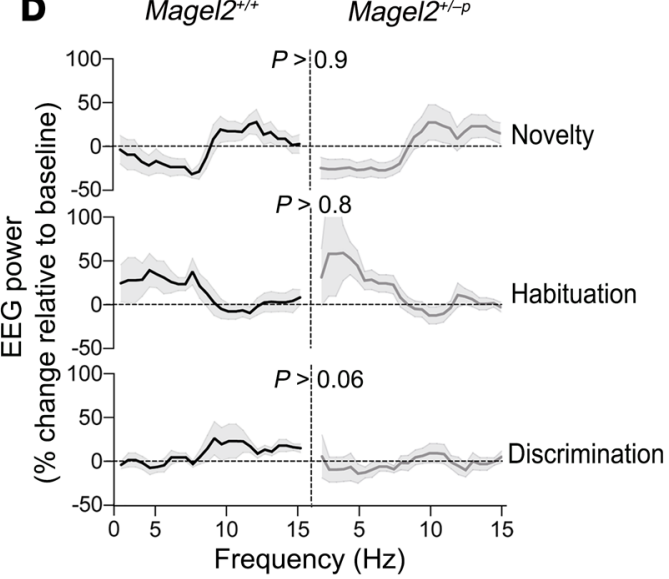

C

- Magel2 $2^{+/+} \quad$ Magel2 $2^{+1-p}$

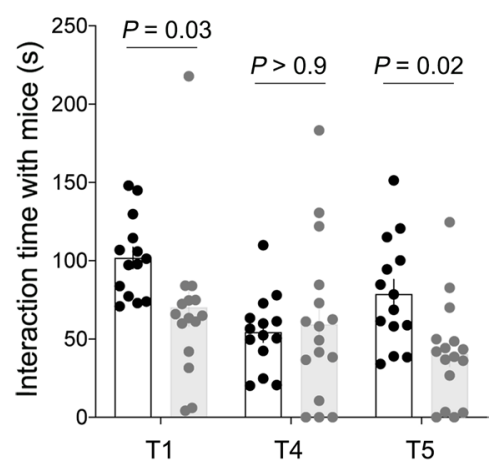

$\mathbf{E}$

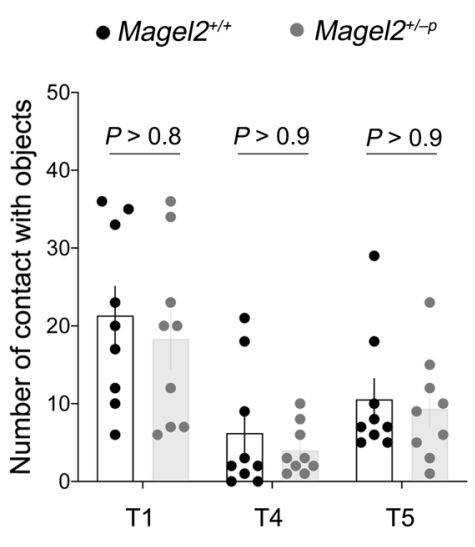

Figure 1. Mage/2 deficiency impairs electrophysiological and behavioral responses to social stimuli. (A) Experimental paradigm. Mice are implanted with EEG electrodes on the skull to record electrophysiological activity during exploration of juveniles (novel or familiar) or objects (novel or familiar). (B) Change

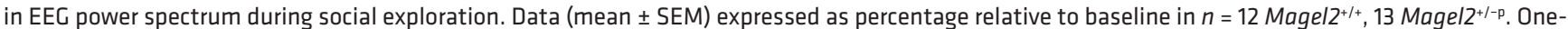
way ANOVA: effect of trials on each group $F_{5,125}=16.47, P<0.0001$; post hoc Sidak test. (C) Time exploring a mouse. Data (mean \pm SEM) in $n=14$ Magel2 $2^{+/+}$, 16 Magel $2^{+/-p}$ mice. Two-way ANOVA: genotype $F_{1,28}=2.55, P=0.001$; social trials $F_{2,56}=6.82, P=0.002$; genotype $\times$ social trials $F_{2,56}=4.69, P=0.01 ;$ post hoc Sidak test. (D) Change in EEG power spectrum during nonsocial exploration. Data (mean \pm SEM) expressed as a percentage relative to baseline in $n=$ $8 \mathrm{Magel}^{+/+}$, $8 \mathrm{Mage} / 2^{+/-p}$. One-way ANOVA: effect of trials on each group $F_{5,125}=5.69, P<0.0001$, post hoc Sidak test. (E) Time exploring an object. Data (mean $\pm \mathrm{SEM}$ ) in $n=9 \mathrm{Magel}^{+/+}, 9 \mathrm{Magel2^{+/-p }}$ mice. Two-way ANOVA: genotype $F_{1,16}=0.4, P=0.5$; object trials $F_{2,32}=28.33, P<0.0001$; genotype $\times$ object trials $F_{2,32}=0.09, P=0.9$.

AVPRs only (30 nM). First, injection of AVP for 9 minutes in freely behaving mice implanted with EEG electrodes (Figure 5A) increased the EEG power spectrum in the theta band during the first 10 minutes, an effect that persisted for 60 minutes (Figure 5B). For comparison, injection of $\mathrm{NaCl}$ vehicle had no effect.

Second, the injection of AVP for 9 minutes starting 5 minutes before social novelty in Magel ${ }^{+/-p}$ mice increased social discrimination later (Figure 5C). For comparison, injection of $\mathrm{NaCl}$ vehicle had no effect. EEG power spectral analysis showed an increase of activity in the theta band that persisted beyond the injection of AVP (Figure 5D).

Third, injection of Manning compound for 9 minutes starting 5 minutes before social novelty in $\mathrm{Magel}^{+/+}$mice decreased social novelty and discrimination (Figure 5E). For comparison, injection of the $\mathrm{NaCl}$ vehicle had no effect. EEG power spectral analysis showed a decrease of activity in the theta band at social novel- ty and an increase of activity at discrimination compared with $\mathrm{NaCl}$-injected controls (Figure 5D).

The source of AVP in the dorsal LS during social behavior. Magel2 mutations impair neuropeptide production and release in human and mouse neurons $(15,31)$, indicating that it could modify the septal content of AVP during behavior, which is normally cued to social novelty (32). Based on the model of synaptic release of OXT in the brain (33), we assumed axonal secretions of AVP in the LS. The effects of Magel2 mutation were assessed on the innervation and experience-dependent activation of AVP neurons. We used neurophysin II antibodies to map AVP projections in the LS (Figure 6A). Fewer and shorter AVP fibers were observed in the dorsal LS of Magel2 ${ }^{+/-\mathrm{p}}$ mice compared with Magel2 $2^{+/+}$controls (Figure 6B). Moreover, we used c-Fos mapping in AVP neurons to determine where and when these neurons activated during social behavior (Figure 6C). AVP neurons were activated from the first social 

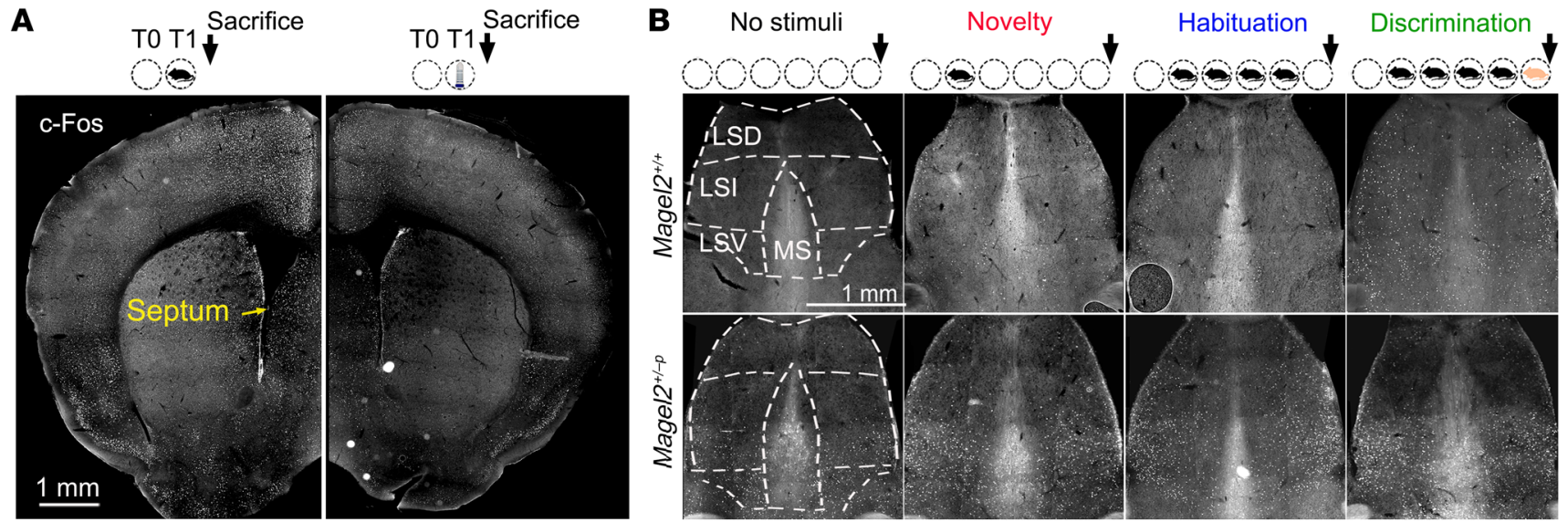

C c-fos cells $/ \mathrm{mm}^{2}$ in Magel2 ${ }^{+/+}$mice
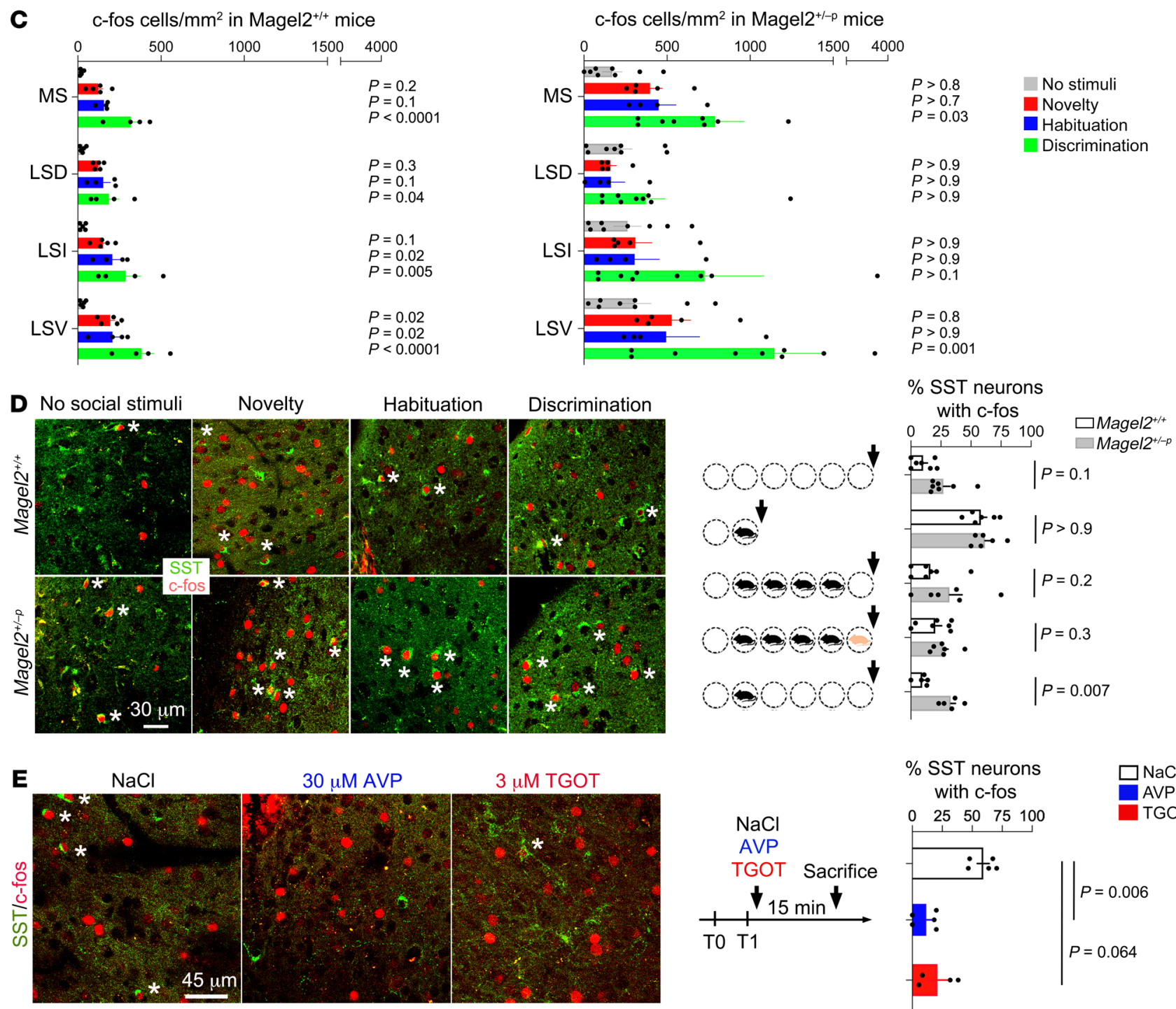
Figure 2. Magel2 deficiency increases c-Fos expression in SST neurons of the dorsal LS during social exploration. (A) c-Fos induction representative of $n=5 \mathrm{Magel}^{+/+}$mouse interacting with a new conspecific for 5 minutes, euthanized 15 minutes later (left) compared with $5 \mathrm{Magel}^{+/++}$mice interacting with a new object in the same conditions (right). (B) c-Fos induction in the septum representative of the mice making 0 to 5 social trials analyzed in panel C. All mice were euthanized 15 minutes after the last trial. (C) Number of c-Fos cells $/ \mathrm{mm}^{2}$ in a total of $28,072 \mathrm{Magel}^{+/+}$and 38,534 Magel $2^{+/-p}$ cells. Mean \pm SEM of $n=5$ controls, 5 novelty, 4 habituation, 4 discrimination Magel2 ${ }^{+/+}$and 7, 5, 4, $9 \mathrm{Magel}^{+/ / p}$ mice, respectively. Twoway ANOVA for social trials in Magel2 ${ }^{+/+} F_{3,56}=31.7, P<0.0001$; in Magel2 $2^{+/-p}$ $F_{3,88}=6.871, P=0.0003$; septal regions in Magel $2^{+/+} F_{3,56}=2.95, P=0.04$; and in Mage/2+/-p $F_{3,56}=2.39, P=0.07$; post hoc Dunnett test results as indicated. Multiple comparison between genotypes by Wilcoxon unpaired $t$ test at novelty in medial septum (MS) $t(8)=3.5, P=0.007$ and LSV $t(8)=$ 2.8, $P=0.02$; at habituation in MS $t(6)=2.76, P=0.032$. (D) Percentage of SST cells with c-Fos expression in the LSD of Magel $^{+/+}$and Magel2 ${ }^{+/-p}$ mice (stars mark double-positive cells). Mean \pm SEM of $n=7$ controls, 6 novelty, 7 habituation, 7 discrimination, 5 novelty +2 hours for Magel2 ${ }^{+/+}$and 7, 5, 6, 6,5 for Mage/2+/-p mice, respectively. Two-way ANOVA for genotype $F_{1,52}=$ 14.05, $P=0.0004$; trials $F_{4,52}=18.36, P<0.0001$; post hoc Sidak test results as indicated. Arrows mark euthanization 15 minutes after the last trial. (E) Percentage of SST cells with c-Fos expression in the LSD of Mage/2 ${ }^{+/+}$ mice (stars mark double-positive cells). Mean \pm SEM of $n=5 \mathrm{NaCl}, 5 \mathrm{AVP}$, 4 TCOT mice injected in dorsal LS via bilateral cannula and euthanized 15 minutes later. Kruskal-Wallis test, $P=0.0012$, post hoc Dunn test results as indicated. LSD, lateral septum dorsal; LSV, lateral septum ventral.

encounter in the paraventricular hypothalamic nucleus (PVN) of Magel2 ${ }^{+/+}$mice and in the bed nucleus of stria terminalis (BNST) of Magel2 ${ }^{+/-\mathrm{p}}$ mice (Figure 6D).

Optogenetic stimulation of AVP neuronal projections in the dorsal LS. The effects of Magel2 mutation on neurophysiology and behavior were assessed upon activation of AVP neuronal projections in the dorsal LS specifically from the BNST or the PVN. For this, Magel2 ${ }^{+/-p}$ mice were crossed with Avp-Cre mice and further injected in the PVN or BNST with Cre-dependent AAV1-ChR2YFP virus. Optic fibers were implanted bilaterally in the dorsal LS and EEG electrodes implanted atop the cortex (Figure 7A). Expression of ChR2-YFP was efficient and specific in AVP neurons of the PVN or BNST (Figure 7B). Blue light stimulation of the BNST/LS pathway during social novelty promoted social discrimination compared with unstimulated controls (Figure 7C). On the contrary, blue light stimulation of the PVN/LS pathway during social novelty failed to significantly restore social discrimination in Magel2 ${ }^{+/-p}$ mice (Figure 7C).

EEG power spectral analysis showed an increase of activity in the theta band at social novelty and discrimination in Magel2 $^{+/-p}$ mice stimulated in the BNST/LS AVP pathway that was consistent with the behavioral outcome (Figure 7D). Finally, c-Fos expression in SST neurons of the dorsal LS was assessed 15 minutes after social discrimination (time of euthanization). Activation of the BNST/LS pathway significantly reduced c-Fos expression in SST neurons, contrary to the same manipulation in the PVN/LS pathway (Figure 7E).

\section{Discussion}

Here, we demonstrated that Magel2 deficiency impaired AVP signaling in the dorsal LS, affecting the adaptive neurophysiological and behavioral functions necessary for discriminative social exploration in adult male mice. This was due to inappropriate innervation of the dorsal LS by AVP neurons, resulting in a sustained activation of SST neurons cued to social novelty. The correction of AVP signaling in the dorsal LS at the time of social novelty restored the neurophysiological and behavioral defects of Magel2 ${ }^{+/-p}$ mice. The findings promote SST neurons as an important entry point to target social disabilities of Magel2 $^{+/-p}$ mice and test new strategies of pharmacological intervention.

A lack of inhibitory tone in the hippocampus of mice lacking OXTR was associated with social and cognitive disabilities that reverted with i.c.v. injection of AVP or OXT, acting on AVPR1A subtypes (34). The origin of abnormalities at GABAergic synapses in $\mathrm{Oxtr}^{-1}$ mice is unknown, but the phenotype is treatable with pharmacological intervention. This is consistent with the AVP injection in the dorsal LS of Magel2 ${ }^{+/-p}$ mice that suppressed SST neuron activation cued to social novelty. Such an effect is associated with the facilitation of GABAergic inhibitory synaptic events to inhibit tonic firing of dorsal LS neurons. SST neurons are the most abundant GABAergic neurons of dorsal LS (http://mouse. brain-map.org/experiment/show/1001). Therefore, the lack of AVP signaling in the dorsal LS of Magel2 ${ }^{+/-p}$ caused by abnormalities in AVP-containing axonal fibers and AVPR density in SST cells would disinhibit their first-order projection neurons. Indeed, Magel2 mutation increased c-Fos expression, basal and experience-dependent, in subfields of the septum where dorsal LS neurons, notably the SST type, send projections (http://connectivity. brain-map.org/ and ref. 35).

SST neurons in the dorsal LS receive glutamatergic synaptic inputs from the hippocampus, which controls mobility in the context of fear (36), and GABAergic inputs facilitated by AVP, which would control social exploration in a nonthreatening context. It is possible that contextual fear (nonsocial) exploits the CA3-LS projections to activate SST neurons, and that social stimuli exploit the PVN/LS AVP pathway to increase the inhibitory tone on SST neurons. Magel2 deficiency impaired AVP-mediated neuromodulation of SST neurons in the dorsal LS, resulting in ectopic c-Fos expression at baseline and in the context of social behavior. Magel2 ${ }^{+/-p}$ male mice exploited AVP neurons of the BNST/LS pathway during social novelty in a nonthreatening context (interactions with samesex juveniles); this pathway is involved in defensive and aggression behaviors in healthy male mice $(37,38)$. Threats, unpredictability, and social anxiety activate the BNST (39), in which the lesion of AVP cells specifically reduced social investigation with other adult males (40). The BNST/LS AVP pathway is larger in male rodents, and regulated by androgens, which could explain the sex difference in social behaviors (41). Consistently, Magel2 ${ }^{+/-p}$ female mice exhibited normal social exploratory behavior (14). Therefore, it is possible that sex differences in the BNST (anatomical, molecular, and behavioral) play a role in the distinct prevalence rates of social disabilities between men and women.

Despite their social disabilities, Magel2 ${ }^{+/-p}$ mice performed well with object discrimination, and theta oscillations were normal in a nonsocial context. In agreement, the sensitivity for discriminating faces and objects is reportedly impaired and enhanced, respectively, in adolescents with ASD (42), which adds to the face validity of the Magel2 $^{+/-p}$ mouse as a model of ASD. Abnormal functions of SST neurons have been reported in other models of ASD. 

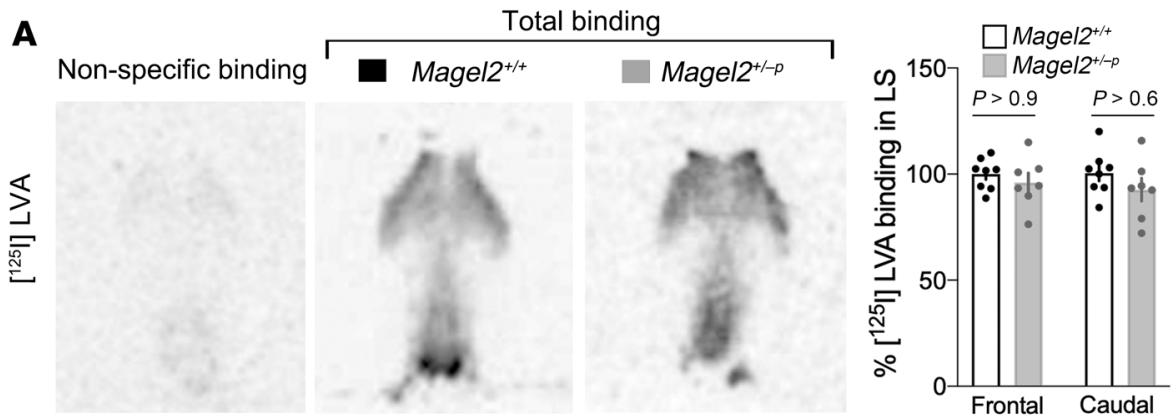

B

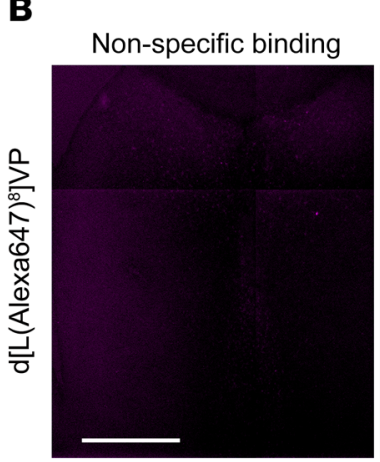

Total binding

for AVPR+OXTR
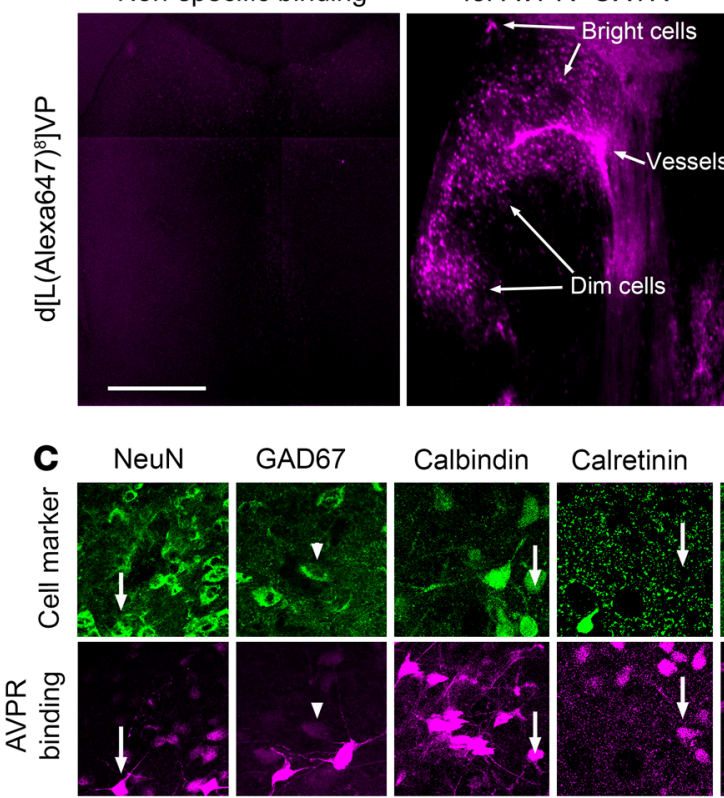

Calretinin
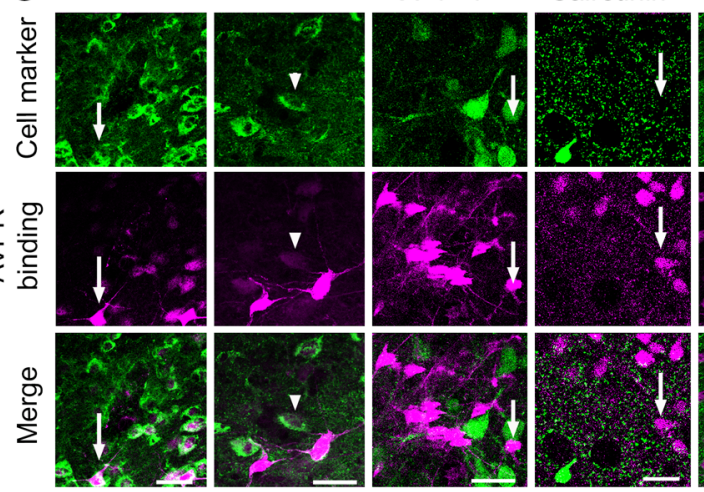

$100 \%$

$69.2 \%$

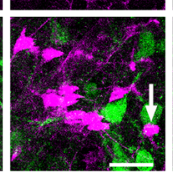

$0.05 \%$
Specific binding
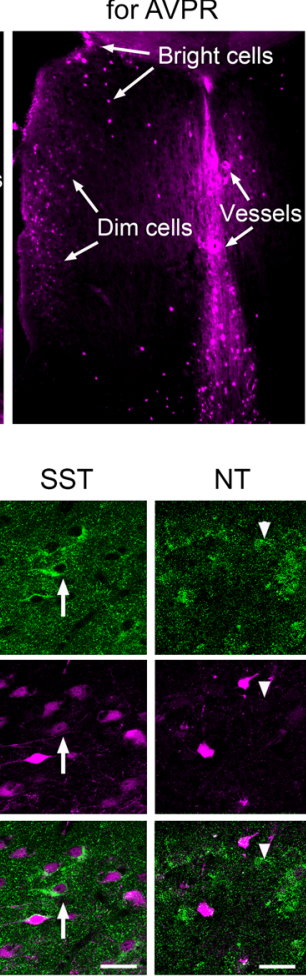

$36 \%$

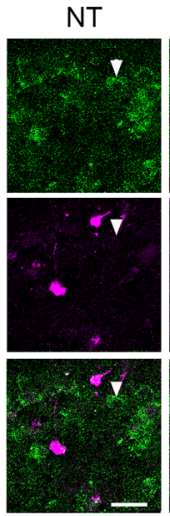

$9.1 \%$
Figure 3. Magel2 deficiency decreases AVP receptor levels in SST neurons of the dorsal LS. (A) Regional precision of $1 \mathrm{nM}$ [25]]LVA binding without (total) and with (nonspecific) unlabeled AVP in excess on frozen brain sections of Magel2+/+ and Magel2+/-p mice. Data (mean \pm SEM) expressed as a percentage relative to Mage/2+/+ in $n=8 \mathrm{Magel} 2^{+/+}$and 7 Magel $2^{+1-p}$ mice. Two-way ANOVA: genotype $F_{1,26}=2.08, P=0.16$; effect of subregions in LS $F_{1,26}=0.13, P=0.71$; post hoc Sidak test for comparisons. (B) Cellular precision of $50 \mu \mathrm{M}$ $\mathrm{d}\left[\right.$ Lys(Alexa Fluor 647) ${ }^{8}$ ]VP binding without (total, $n=4$ mice) and with (nonspecific, $n=$ 3 mice) $100 \mu \mathrm{M}$ Manning compound injected directly into the LS of Mage/2 ${ }^{+/+}$mice. OXTR competitor ( $5 \mu \mathrm{M}$ TCOT, $n=5$ mice) coinjected with d[Lys(Alexa Fluor 647) 8 ]VP to specify AVPR binding sites representative of 3 independent experiments. Scale bar: $1 \mathrm{~mm}$. Binding affinity for AVPR subtypes presented in Supplemental Table 1. (C) Coexpression of AVPR binding sites with the indicated markers. Arrows point to cells with "bright" labeling and arrowheads to cells with "dim" labeling. Scale bars: $25 \mu \mathrm{m}$. Percentage of cells coexpressing AVPR and cell markers are indicated. (D) Proportion of cell types with AVPR binding sites for d[Lys(Alexa Fluor 647$)^{8}$ ]VP in dorsal LS. Data (mean \pm SEM) expressed as percentage relative to $\mathrm{Magel}^{+/+}$in $n=6 \mathrm{Magel}^{+/+}$and $6 \mathrm{Magel}^{+/ /-p}$ mice. Three-way ANOVA for cell type $F_{2,59}=$ 37.27, $P<0.0001$; AVPR binding $F_{1.59}=49.85$, $P<0.0001$; genotype $F_{1,59}=1.83, P=0.18$; cell type $\times$ AVPR binding $\times$ genotype $F_{2,59}=5.35$, $P=0.0073$; post hoc Sidak test results as indicated.

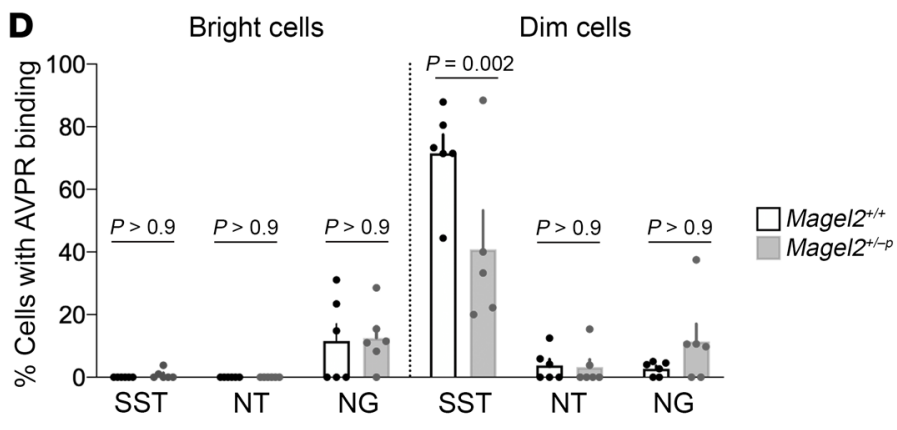

For example, Mecp2 deletion restricted in SST neurons caused seizures and stereotypies (43), while Pten deletion impaired the cortical ratio of parvalbumin/SST cells, altering brain EEG activity and social behavior (44). Previous studies recording local field potentials in the visual cortex reported that SST neurons drive low-frequency band oscillations (45). Here, we showed that AVP injection in the dorsal LS as well as social novelty increased theta oscillations (4-12 Hz), whereas Magel2 mutation as well as AVPR antagonist injection in the dorsal LS decreased such activity. This is consistent with the modulation of theta rhythm $(3-10 \mathrm{~Hz})$ across multiple regions of the social brain network during exploration of juveniles (24). What's more, AVP-deficient Brattleboro rats centrally administered with AVP corrected for several hours the frequency deficit of theta rhythm (46), which is consistent with our finding that AVP administration restored social discrimination more than 1 hour after the injection. 
A
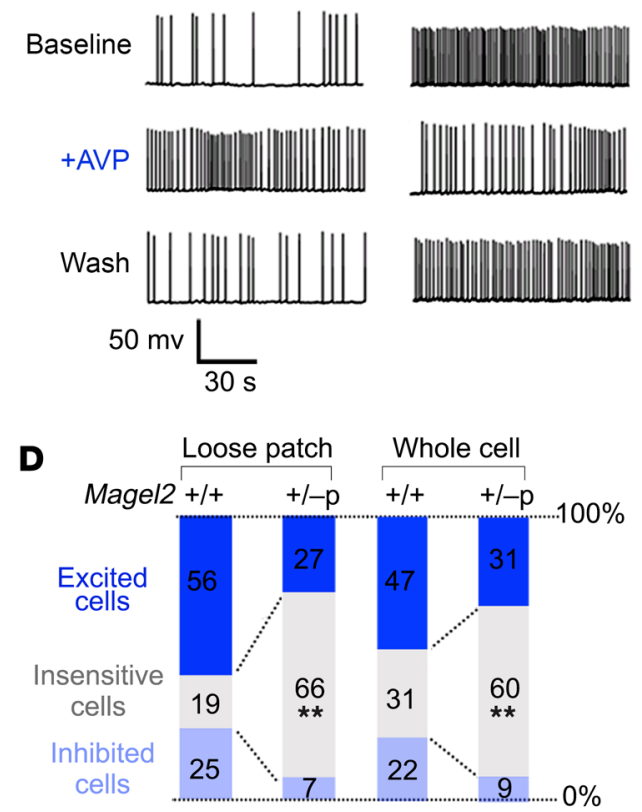

B

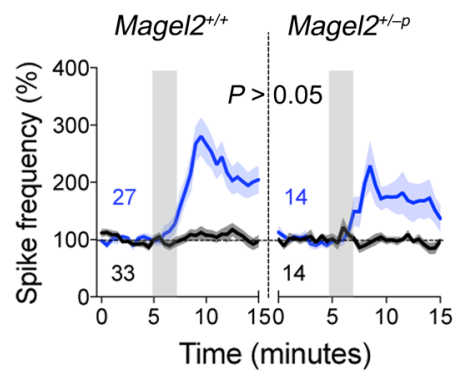

C

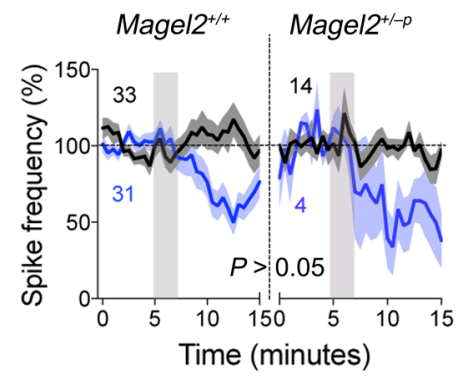

E Cadaverine dye in

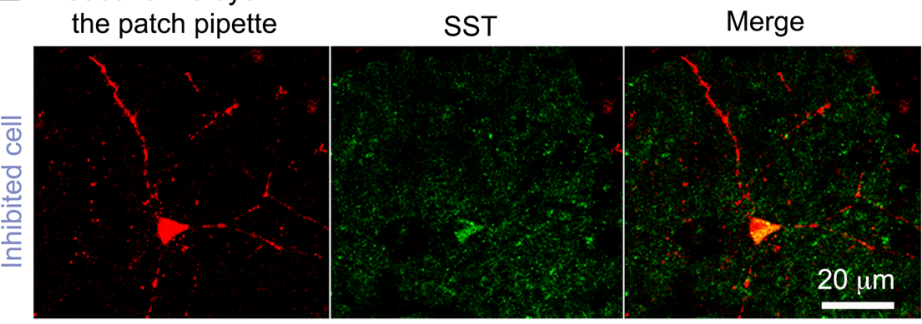

$\mathbf{F}$ Excited cells

Inhibited cells

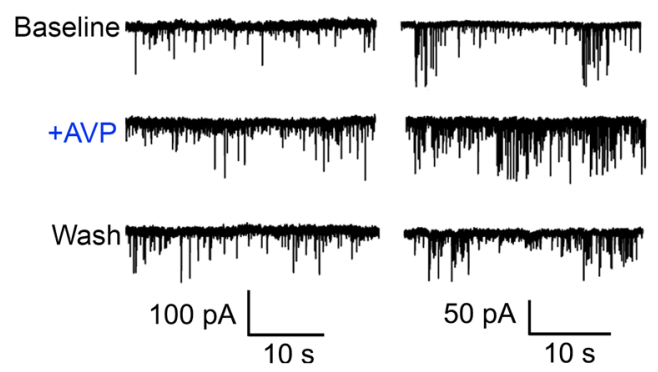

Figure 4. Magel2 deficiency reduces neuronal response to AVP in the dorsal LS. (A) Typical firing patterns of dorsal LS neurons in coronal slices at baseline and upon 2-minute bath application of $1 \mu \mathrm{M}$ AVP (whole cell recordings). Responses representative of more than 5 experiments reverted to baseline after washout. (B) Frequency of action potentials in AVP-excited cells. Mean \pm SEM expressed as percentage relative to baseline prior to AVP stimulation (gray bar). Numbers indicate cells excited (blue) and insensitive to AVP (black). Three-way ANOVA for time $F_{30,2259}=9.5, P<0.0001 ;$ AVP $F_{1,76}=16.9, P<0.0001$; genotype $F_{1,76}=1.2, P>0.2$; time $\times$ AVP $F_{30,2259}=8.6, P<0.0001$; time $\times$ AVP $\times$ genotype $F_{30,2259}=1.04, P=0.4$; post hoc Tukey test. (C) Frequency of action potentials in AVP-inhibited cells. Mean \pm SEM expressed as percentage relative to baseline. Numbers indicate cells excited (blue) and insensitive to AVP (black, same as in panel B). Three-way ANOVA for time $F_{30,2077}=2.55, P<0.0001 ;$ AVP $F_{1,71}=4.9, P=0.03$; genotype $F_{1,71}=$ $0.6, P>0.4$; time $\times$ AVP $F_{30,2077}=3.05, P<0.0001$; time $\times$ AVP $\times$ genotype $F_{30,2077}=0.4, P>0.9$; post hoc Tukey test. (D) Proportion of cells categorized according to their responses to AVP on the frequency of action potentials recorded in loose patch $\left(n=32 \mathrm{Magel2^{+/+ }}\right.$, $26 \mathrm{Magel2^{+/-p }}$ cells $)$ or whole cell

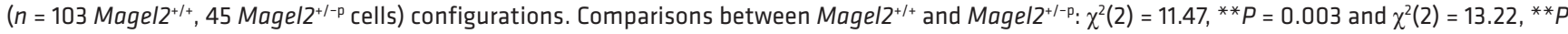
$=0.0013$. (E) AVP-inhibited cell representative of at least 2 independent experiments marked with cadaverine dye injected in the patch pipette and labeled with SST antibodies. (F) Effect of AVP on the frequency of synaptic events in dorsal LS neurons from Mage/2 ${ }^{+/+}$mice representative of more than 5 experiments. Effect sizes are presented in Table 1.

In humans, intranasal administration of AVP increased activity in the LS and reciprocated social collaboration (47). Intranasal OXT administration enhances the suppression of oscillatory activity (8-25 Hz) during execution and observation of social actions (48). Altogether, OXT- and AVP-dependent modulation of neural activity in response to social stimuli directly affect EEG activity, which may have a predictive value for the impact of such treatment in ASD-associated disorders. Furthermore, an imbalance between inhibition and excitation is associated with ASD, and AVP treatment could reset the balance by altering the functions of SST neurons (49).
This study focused on Magel2-KO mice that cannot model the gain of function of the C.1996dup mutation reported in the most severe cases of SYS (50). The point mutations in MAGEL2 causing SYS should result in the production of truncated proteins, whereas it is the deletion of MAGEL2 and other genes located in the imprinted locus that cause PWS. Both syndromes share a similar phenotype in infancy and autism that is more frequent in SYS. Very likely, SYS might be caused by the loss of normal function of MAGEL2 and gain of abnormal function of the truncated protein resulting from the point mutation. Thus, using a mouse model, our study reports the loss of function of Magel2 present in 


\section{Table 1. Frequency of excitatory and inhibitory synaptic events in dorsal LS neurons of Magel2+/+ mice}

\begin{tabular}{lcccc} 
Frequency & Excited cells & \multicolumn{3}{c}{ Inhibited cells } \\
EPSC basal (Hz) & $7.8 \pm 1.5(100 \%)$ & $n=40$ & $17 \pm 1.2(100 \%)$ & $n=21$ \\
+ + AVP (\% basal) & $122 \pm 12$ & $n=18$ & $78 \pm 9$ & $n=7$ \\
+ TTX (\% basal) & $32 \pm 6$ & $n=6$ & $99 \pm 12$ & $n=4$ \\
+ AVP + TTX (\% basal) & $66 \pm 9.9$ & $n=6$ & $135 \pm 26$ & $n=3$ \\
+ CNQX (\% basal) & $305 \pm 16^{\mathrm{C}}$ & $n=6$ & $7 \pm 3^{\mathrm{C}}$ & $n=4$ \\
+ AVP + CNQX (\% basal) & $159 \pm 10^{\mathrm{A}}$ & $n=4$ & $76 \pm 62$ & $n=3$ \\
IPSC basal (Hz) & $48 \pm 15(100 \%)$ & $n=26$ & $61 \pm 19(100 \%)$ & $n=13$ \\
+ AVP (\% basal) & $236 \pm 6.8^{\mathrm{C}}$ & $n=16$ & $224 \pm 9^{\mathrm{A}}$ & $n=6$ \\
+ TTX (\% basal) & $19 \pm 6$ & $n=7$ & $36 \pm 5$ & $n=4$ \\
+ AVP + TTX (\% basal) & $93 \pm 35^{\mathrm{A}}$ & $n=4$ & $62 \pm 15^{\mathrm{A}}$ & $n=3$ \\
IPSC + EPSC basal (Hz) & $56 \pm 15(100 \%)$ & $n=52$ & $78 \pm 20(100 \%)$ & $n=30$ \\
+ AVP (\% basal) & $261 \pm 44^{\mathrm{B}}$ & $n=14$ & $374 \pm 97^{\mathrm{C}}$ & $n=10$ \\
+ TTX (\% basal) & $8.5 \pm 6$ & $n=7$ & $23 \pm 4.5$ & $n=6$ \\
+ GABAzine (\% basal) & $23 \pm 1$ & $n=16$ & $30 \pm 10$ & $n=8$ \\
+ GABAzine + AVP (\% basal) & $24 \pm 4.7^{\mathrm{C}}$ & $n=15$ & $13 \pm 6^{\mathrm{C}}$ & $n=6$
\end{tabular}

Frequency of excitatory and inhibitory synaptic events (mean \pm SEM, $n$ cells as indicated) in dorsal LS neurons of Mage/2+/+ mice. Effect of AVP ( $1 \mu \mathrm{M}, 2$ minutes) tested with and without the sodium channel blocker TTX $(30 \mu \mathrm{M})$, GABA-A receptor antagonist GABAzine $(6 \mu \mathrm{M})$, AMPA receptor antagonist CNQX $(1 \mu \mathrm{M})$. One-way ANOVA with post hoc Sidak test comparing basal versus AVP or TTX or CNQX or CABAzine, and AVP versus AVP + CNQX or AVP + TTX or AVP + CABAzine. IPSC, inhibitory postsynaptic current; EPSC, excitatory postsynaptic current. ${ }^{A} P<0.05 .{ }^{B} P<0.01 .{ }^{C} P<0.001$.

to habituate to the arena. After 20 minutes of basal activity, a juvenile male conspecific (3-6 weeks old) was introduced in the box for 5 minutes. The same presentation happened 4 times at 20-minute intervals to habituate the mice with one another. After another 20 minutes, a new male juvenile different from the first one was introduced in the box for 5 minutes. The setup was systematically cleaned using disinfectant wipes before a new experimental mouse was challenged. This test in rodents typically decreases exploration of the known juvenile while it increases exploration of the new juvenile. For nonsocial behavior, a similar protocol was used except that an object (Falcon tube or Lego) was introduced into the arena. Tests were video recorded to extract the time spent sniffing a juvenile during each trial (nose of the experimental animal in contact with sniff holes in the cup) or the number of times the object was contacted (nose in contact with the object). All procedures on animals were performed between 800 and 1500 hours (during the light phase) according to the Animal Research: Reporting of In Vivo Experiments guidelines.

In vivo pharmacology. Surgeries were performed under ketamine $(6.6 \mathrm{~g} / \mathrm{kg})$ and xylazine $(1.3 \mathrm{~g} / \mathrm{kg})$ anesthesia. Bilateral cannula (26 gauge, Phymep) heading to the lateral septum were implanted in mice using a stereotaxic frame (anterioposterior $[\mathrm{AP}]+0.05 \mathrm{~cm}$, mediolateral $[\mathrm{ML}] \pm 0.04 \mathrm{~cm}$, dorsoventral [DV] $-0.3 \mathrm{~cm})$. Dental cement (Paladur, Henry Schein) and sutures were used

both syndromes. Future studies will extend the toolbox of genetic mouse models to study MAGEL2-related diseases.

\section{Methods}

Details of reagents are presented in Supplemental Table 2.

Animals. $\operatorname{Avp}^{\mathrm{tm} 1.1}(\mathrm{Cre})^{\mathrm{Hz}} / \mathrm{J}(\mathrm{Avp}$-Cre $)$ male mice were purchased from The Jackson Laboratory, and Magel2 ${ }^{\text {tm1.1Mus }} / \mathrm{J}$ (Magel2-KO) mice were bred in-house. Magel2 ${ }^{+\mathrm{m} /-\mathrm{p}}$ (paternal KO allele) mice were obtained by crossing WT females with male Magel2 ${ }^{\mathrm{p}} / \mathrm{m}$ (maternal KO allele). Magel 2 being a maternally imprinted gene, Magel ${ }^{+\mathrm{m} /-\mathrm{p}}$ mice did not express Magel2 and were considered functionally KO (referred to as Magel2 ${ }^{+/-p}$ ) as previously described (14). Littermates expressing 2 WT alleles were used as controls (Magel2 ${ }^{+/+}$). Genotyping primers and protocols are as recommended by the manufacturer. All mice were maintained on a C57B6/J background (Janvier Labs). All animals were housed on a 12-hour light/12-hour dark cycle in a specific and opportunistic pathogen-free zone with access to ad libitum food and water. Enrichment consisted of cotton pieces and igloos to build a nest. Animals were group caged until surgeries. After implantation surgery, animals were isolated until the end of the experiments.

Behavior. The effects of Magel2 ${ }^{+/-p}$ mutation were evaluated in a social habituation/discrimination task as described previously (51). A week before the first experiment, male mice (3-4 months old) were habituated to manipulation by the experimenter. A plexiglass transparent circular arena ( $24 \mathrm{~cm}$ diameter) was video recorded for the time of the experiment and accommodated with clean litter, a water bottle, and food before the beginning of the experiment. For social behavior, a transparent box with sniff holes was also introduced before the beginning of the experiment and a test mouse was left for 10 minutes to secure the implant. Animals were given antiinflammatory medication (doliprane, $6 \mathrm{mg}$ ) and were monitored daily and allowed to recover for 14 days after surgery. Positions of cannulas were verified a posteriori. Drugs were injected through the cannula using bilateral injector connected through 2 different tubes to two $1 \mu \mathrm{L}$ Hamilton syringes controlled by microinjector pumps (micro 4, World Precision Instruments). Injections of $900 \mu \mathrm{L} /$ hemisphere were performed at $100 \mathrm{~nL} / \mathrm{min}$ and diffusion perimeter was estimated by injection of Alexa Flour 594 cadaverine (Life Technology, Thermo Fisher Scientific, $50 \mu \mathrm{M}$, molecular weight comparable to the peptides used) using the same parameters just before euthanasia. The injection setup was connected to the cannula 5 minutes before the injection and left for 5 minutes after the end of the injection, such that it overlapped with the interaction with a mouse or object. For behavioral experiments, injections were started 5 minutes before the introduction of the stimulus (object or juvenile). Mice were allowed at least 5 days between consecutive injections of $\mathrm{NaCl} 0.9 \%$, Manning compound (10 nM, Merck), or AVP ( $1 \mu \mathrm{M}$, Merck) performed in random order. Concentrations of aforementioned selective ligands were determined based on published selectivity profiles on mouse receptors (30).

Optogenetics. At 4 weeks, Avp-Cre Magel2 ${ }^{+/-p}$ animals were injected with AAV1 (EF1a::DIO-ChR2-eYFP;WPRE::hGH from viral vector core, University of Pennsylvania, Philadelphia, Pennsylvania, USA) $\left(500 \mathrm{~nL} /\right.$ hemisphere, $2 \times 10^{11}$ viruses $/ \mathrm{mL}$ ) either in PVN (AP -0.01 $\mathrm{cm}, \mathrm{ML} \pm 0.02 \mathrm{~cm}, \mathrm{DV} 0.48 \mathrm{~cm}$ ) or in BNST (AP $0.05 \mathrm{~cm}, \mathrm{ML} \pm 0.1 \mathrm{~cm}$, DV $-0.4 \mathrm{~cm}$ ) using a microinjector-controlled syringe (micro-4, World Precision Instruments) and needle (Nanofil 33G beveled needle, World Precision Instruments). One month after the infection, bilateral optic fibers (dual fiberoptic cannula, Doric lenses, $0.53 \mathrm{NA}$ ) were 
A

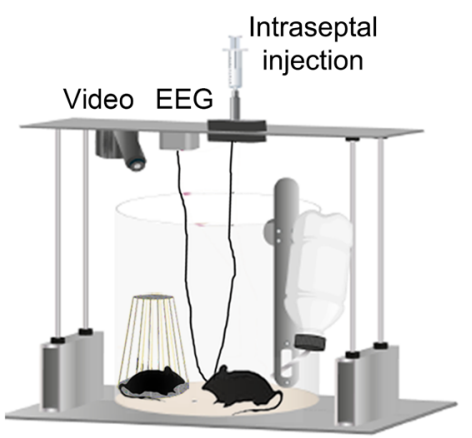

C

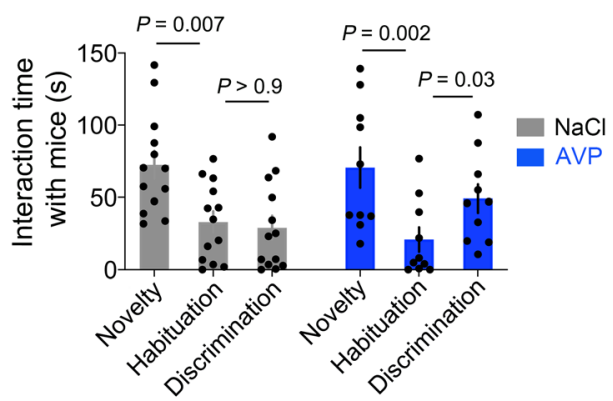

B

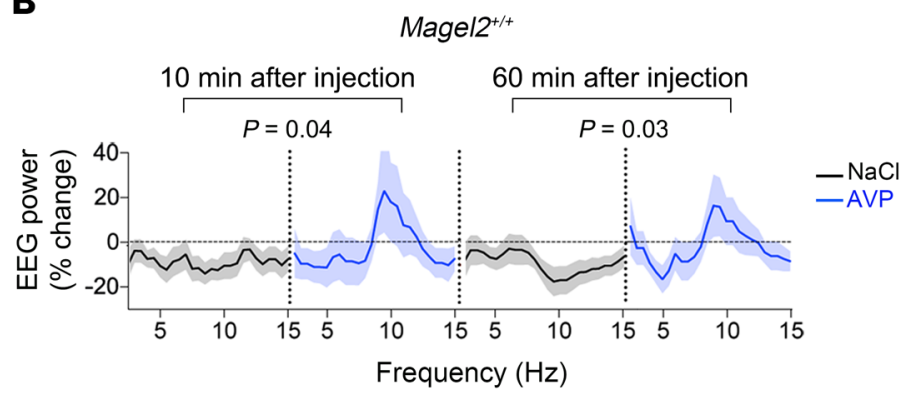

D $\quad$ Magel2 $2^{t-p}$

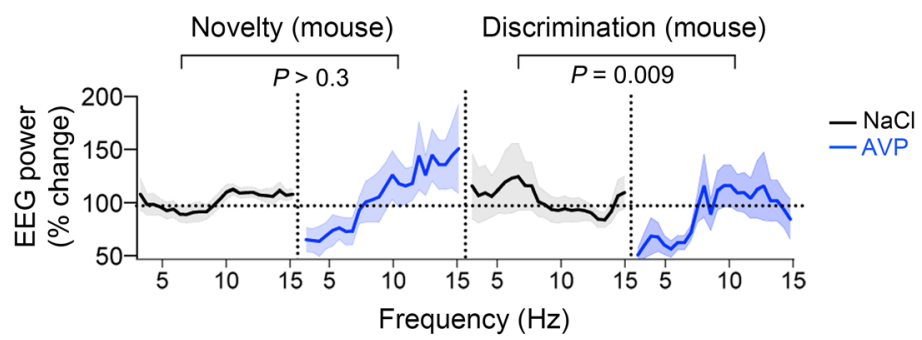

$\mathbf{F}$

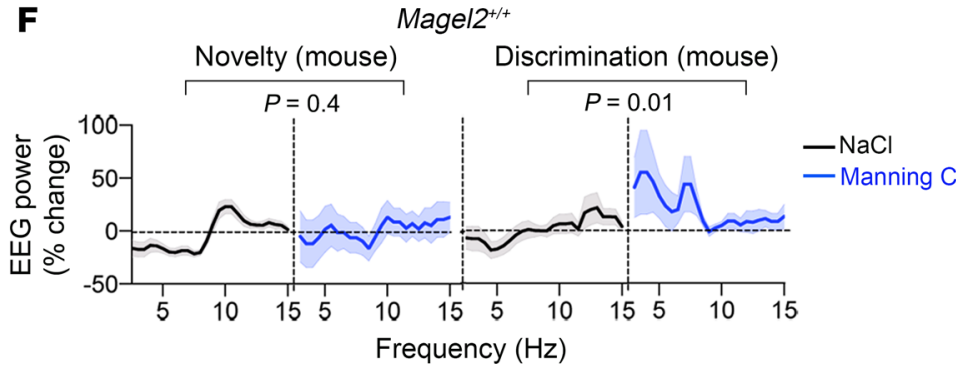

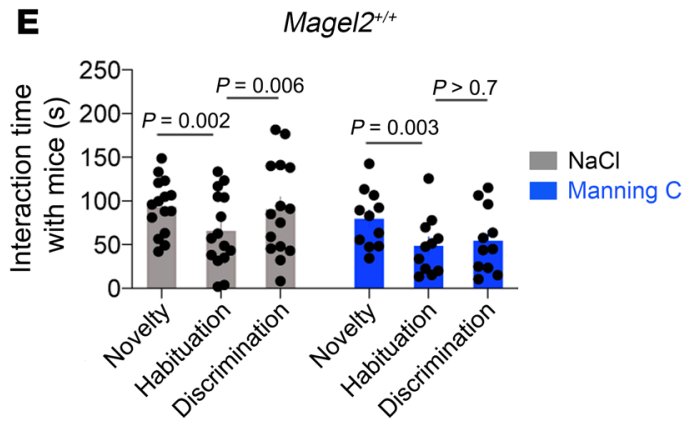

Figure 5. Restoration of social discrimination in Magel2 ${ }^{+/-p}$ mice by AVP injection in the dorsal LS. (A) Experimental setup. (B) Change in EEG power spectrum with intraseptal injection of AVP (30 $\mu \mathrm{M}$ for 9 minutes starting 5 minutes before the first social trial) in Magel2 ${ }^{+/+}$mice. Data (mean \pm SEM) of $n=13 \mathrm{NaCl}$ and 10 AVP. Two-way ANOVA for AVP for 10 minutes: $F_{1,650}=8.13, P=0.004$ and for 60 minutes: $F_{1,650}=15.5, P<0.0001$; post hoc Sidak test for pairwise comparisons between $\mathrm{NaCl}$ and AVP groups as indicated. (C) Time exploring a mouse upon injection of AVP (30 $\mu \mathrm{M}$ for 9 minutes starting 5 minutes before the first social trial) in dorsal LS of Magel2 ${ }^{+/-p}$ mice. Data (mean \pm SEM) in $n=14$ NaCl, 16 AVP-injected mice. Two-way ANOVA for social trials $F_{2,42}=17.99, P<0.0001 ;$ AVP $F_{1,21}=0.03, P=0.8$; post hoc Sidak test comparing consecutive trials as indicated. (D) Change in EEG power spectrum with intraseptal injection of AVP during behavior. Data (mean \pm SEM) of $n=14 \mathrm{NaCl}$ and 9 AVP Mage/2 ${ }^{+/-p}$ mice. Two-way ANOVA for AVP at novelty $F_{23,504}$ $=1.27, P=0.17$ and at discrimination $F_{23,480}=1.74, P=0.018$; post hoc Sidak test for pairwise comparisons between NaCl and AVP groups as indicated. (E) Time exploring a mouse upon injection of Manning compound (MC, $10 \mathrm{nM}$ for 9 minutes starting 5 minutes before the first social trial) in dorsal LS of Magel2 ${ }^{+/+}$mice. Data (mean $\pm \mathrm{SEM}$ ) in $n=15 \mathrm{NaCl}, 11 \mathrm{MC}$ Magel2 ${ }^{+/+}$mice. Two-way ANOVA: social trials $F_{2,48}=11.12, P=0.0001 ; \mathrm{MC} F_{1,24}=2.46, P=0.12$; post hoc Dunnett test for pairwise comparison between consecutive trials as indicated. (F) Change in EEG power spectrum with intraseptal injection of MC during behavior. Data (mean \pm SEM) expressed as percentage relative to baseline in $n=17 \mathrm{NaCl}, 11 \mathrm{MC}$. Two-way ANOVA for social novelty $F_{25,650}=$ 2.83, $P<0.0001$; $M C F_{1,650}=2.56, P=0.1$; social discrimination $F_{25,676}=0.6, P=0.9$; $M C F_{1,676}=31.3, P<0.0001$; post hoc Sidak test for pairwise comparison between $\mathrm{NaCl}$ and $\mathrm{MC}$ groups as indicated.

implanted in the LS (AP $+0.05 \mathrm{~cm}, \mathrm{ML} \pm 0.04 \mathrm{~cm}, \mathrm{DV}-0.3 \mathrm{~cm})$. All surgeries were performed under ketamine $(6.6 \mathrm{~g} / \mathrm{kg})$ and xylazine $(1.3$ $\mathrm{g} / \mathrm{kg}$ ) anesthesia. LEDs were calibrated at $500 \mathrm{~mA}$ to deliver $2 \mathrm{~mW}$ at the tip of the optic fiber, monitored with a luminometer (Thorlabs). Stimulations of channelrhodopsin consisted of $5 \mathrm{~ms}$ pulses, $20 \mathrm{~Hz}$ at $473 \mathrm{~nm}$. Positions of optic fibers were verified a posteriori.

EEG. Mice at least 2 months of age were implanted with surface electrodes atop the somatosensory cortex and allowed 2 weeks of recovery from surgery (52). EEG signals were acquired for 3 hours during interactions with a mouse or an object at $600 \mathrm{~Hz}$, stored, and analyzed using Sirenia (Pinnacle Inc.). Dual video-EEG inspection permitted the exclusion of artifacts associated with scratching, eating, drinking, chewing, self-grooming, and sleep from the period of interest, which included exploration and behavioral interactions with a conspecific or an object. Frequencies above $100 \mathrm{~Hz}$ were filtered and data analyzed with NeuroScore software (Data Sciences International). Frequency bands of $0.5 \mathrm{~Hz}$ were defined, and the power of each of these bands was evaluated for each 10-second epoch composing the signal. Mean values of power for each band of frequency were calculated for the periods relevant to the behavior (while the social 
A

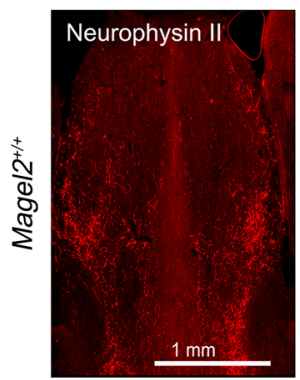

Fiber tracés

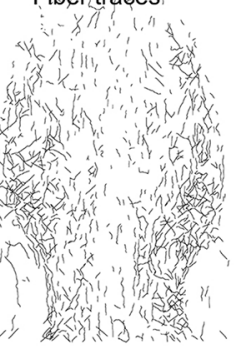

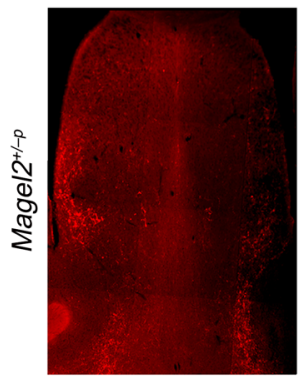

B

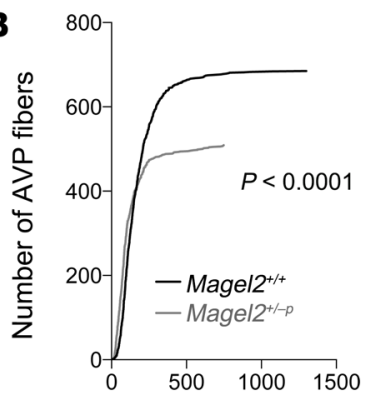

Length of AVP fibers $(\mu \mathrm{m})$

C
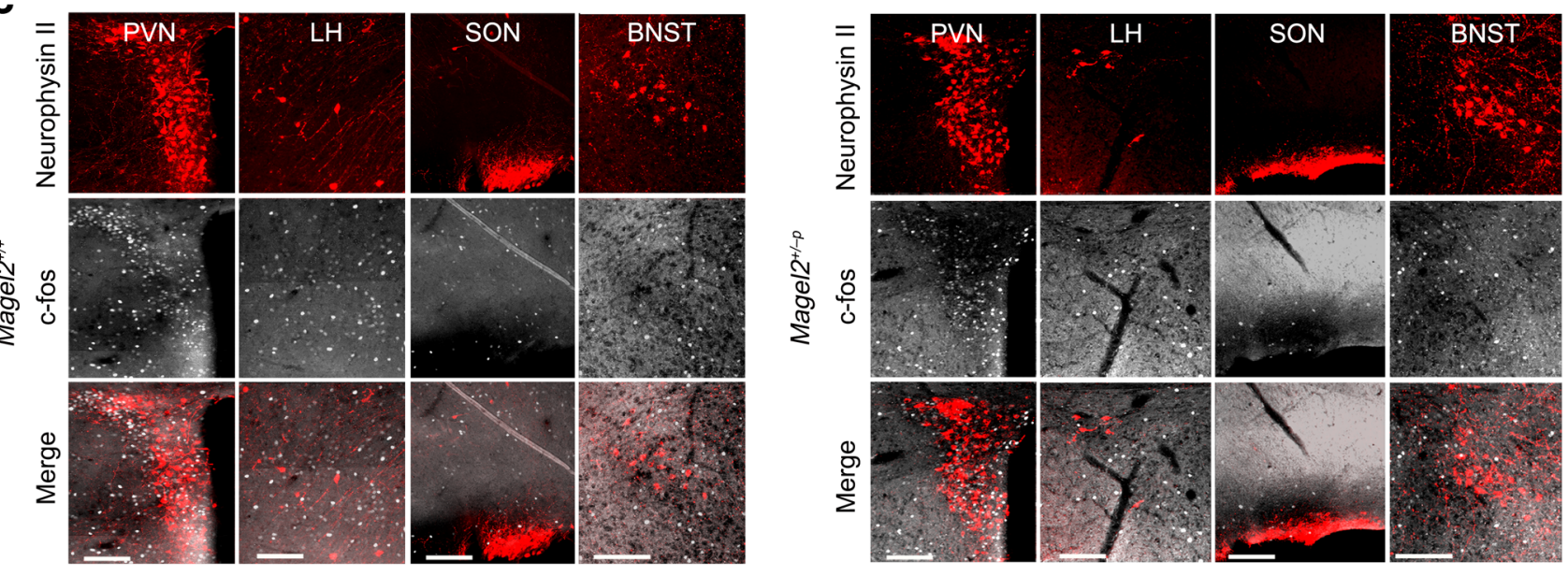

D

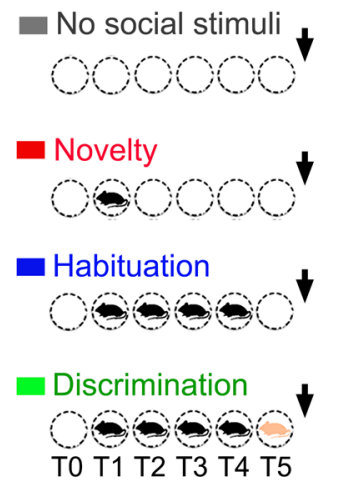

$\%$ AVP neurons with c-fos in Magel2 ${ }^{+/+}$mice

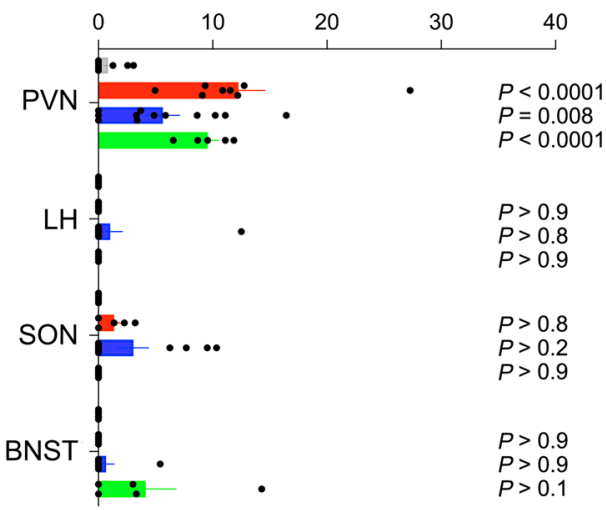

$\%$ AVP neurons with c-fos in Magel2 ${ }^{+/-p}$ mice

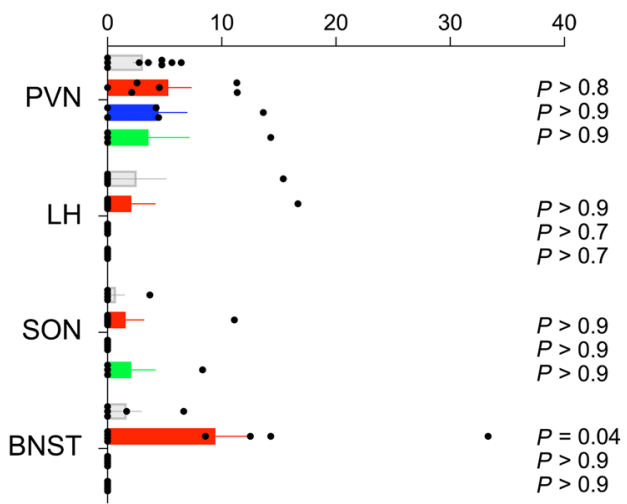

Figure 6. Mage/2 deficiency impairs the number of fibers projecting in the dorsal LS and activation of the PVN AVP neurons by social stimuli. (A) Neurophysin II (AVP) immunoreactivity and semiautomated fiber tracing in the septum representative of the number of mice analyzed in panel B. (B)

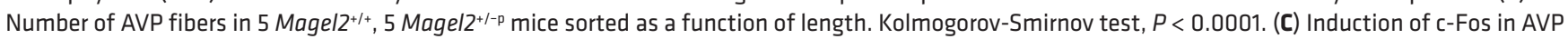
neurons of the paraventricular hypothalamic nucleus (PVN), lateral hypothalamus (LH), supraoptic nucleus (SON), and bed nucleus of stria terminalis (BNST) after social novelty representative of the number of mice analyzed in panel $\mathbf{D}$. Scale bars: $50 \mu \mathrm{m}$. (D) Percentage of AVP neurons expressing c-Fos after social tasks (euthanized 15 minutes after trial 5). Mean \pm SEM of $n=8$ controls, 8 novelty, 12 habituation, 7 discrimination Magel2 ${ }^{+/+}$and $9,8,5,5$ Magel2 $2^{+-p}$ mice, respectively. Two-way ANOVA for social trials in Magel2 ${ }^{+/+} F_{3,96}=4.34, P=0.006$ and in Magel2 ${ }^{+/-p} F_{3,76}=2.07, P=0.11$; regional AVP neurons in Magel2 $2^{+++} F_{3,96}=23.76, P<0.0001$ and in Magel2 $2^{+/-p} F_{3,75}=1.76, P=0.16$; social trials $\times$ regional AVP neurons in Magel2 $2^{+/+} F_{9,96}=4.36, P<0.0001$ and in Magel2 $2^{+-p} F_{9,75}=1.17, P=0.32$; post hoc Dunnett test results as indicated. Multiple comparison between Magel2 $2^{+/+}$and Magel $2^{+/-p}$ by Sidak test in the PVN $t(8)=3.05, P=0.038$ and in the BNST $t(6)=4.5, P=0.0002$ at novelty.

stimulus or the object was present in the arena). To limit the impact of interindividual variability, each frequency band was normalized to a baseline taken from the same experimental animal during the same experiment. For the novelty, data represent the changes in power induced by the presence of the juvenile or the object at trail 1 (T1) for 5 minutes normalized to a 5 -minute period taken before the introduc- tion of the juvenile or the object. For the habituation, data represent the changes in power occurring from $\mathrm{T} 1$ to $\mathrm{T} 4$. For the discrimination, data represent the changes in power occurring from T4 to T5. For the effect of $\mathrm{AVP}$ and $\mathrm{NaCl}$, data represent the changes in power occurring between a baseline period of 10 minutes taken before the drug injection and a 10- or 60-minute period after the injection. 
A

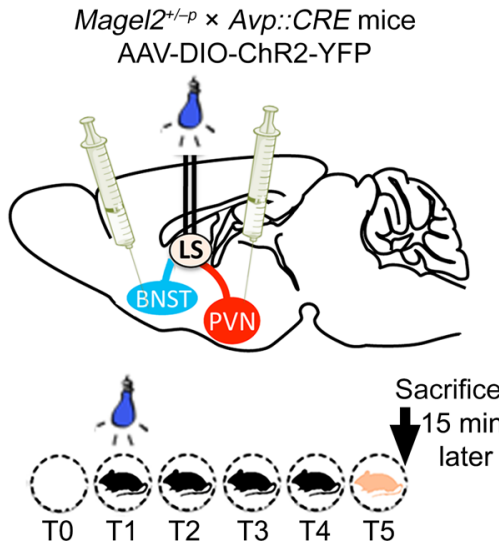

B

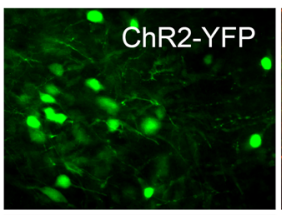

Neurophysin II

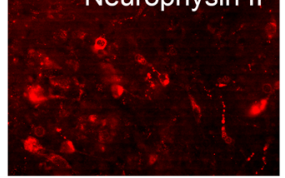

BNST neurons
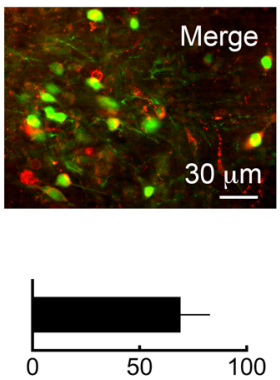

$\%$ Neurons infected

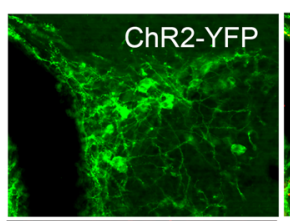

Neurophysin II

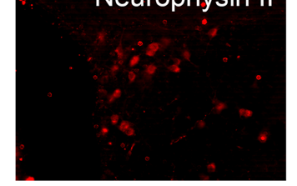

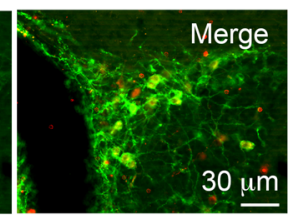

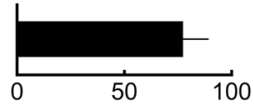

$\%$ Neurons infected
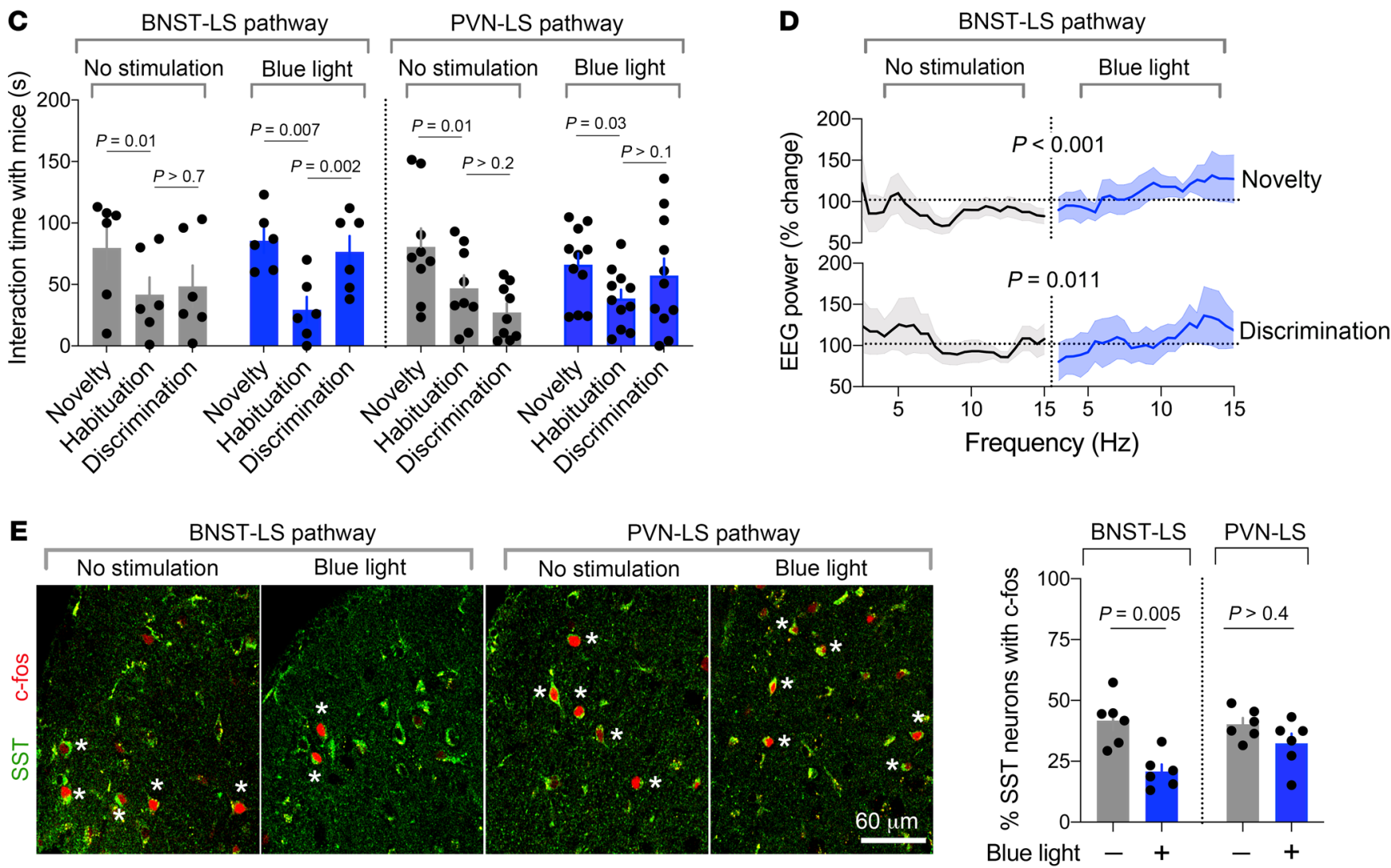

Figure 7. Correction of social disabilities in Mage/2-deficient mice with optogenetic stimulation of AVP neurons in the BNST/LS pathway correlates with the deactivation of SST neurons in the dorsal LS. (A) Viral strategy to express ChR2 in AVP neurons of 2 distinct regions with established projections in the LS where optic fibers were implanted to manipulate AVP responses during social behavior. (B) Expression of Cre-dependent ChR2-YFP in AVP neurons in the BNST or in the PVN of Magel2+/-p mice. Percentage (mean \pm SEM) of AVP neurons expressing ChR2-YFP in BNST or PVN of 6 Mage/2+/-p mice per group. (C) Time exploring a mouse upon stimulation of ChR2 with blue light ( $473 \mathrm{~nm}, 20 \mathrm{~Hz}, 5 \mathrm{~ms}$ pulses for 9 minutes during social novelty) in dorsal LS projections from AVP neurons of the BNST or the PVN in Magel2 ${ }^{+/-p}$ mice. Mean \pm SEM of $n=6$ no light, 6 blue light for the BNST/LS groups and 9 no light, 11 blue light for the PVN/LS groups. Two-way ANOVA for the BNST/LS pathway: effect of social trials $F_{2,10}=17.11, P=0.0006$ and interaction with CHR2 stimulation $F_{2,10}=3.6, P=0.06$; for the PVN/LS pathway: effect of social trials $F_{2,36}=9.17, P=0.0006$ and interaction with ChR2 stimulation $F_{2,36}=4.19, P=0.02$; post hoc Sidak test for pairwise comparisons. (D) Change in EEG power spectrum with intraseptal stimulation of ChR2 during social novelty. Mean \pm SEM expressed as percentage relative to baseline in $n=11$ no light, 6 blue light. Two-way ANOVA for the effect of blue light on frequency at T1 $F_{1,390}=18.27, P<0.0001$ and at T5 $F_{25,400}=1.73, P=0.016$. (E) Percentage of SST neurons expressing c-Fos in dorsal LS 15 minutes after the social discrimination trial (time of euthanization). Stars mark colabeled cells. Mean \pm SEM of 6 Magel2 ${ }^{+/-p}$ mice per group. Kruskal-Wallis test, $P=0.011 ;$ post hoc Dunn test results as indicated.

Slice electrophysiology. Animals (4-8-week-old males) were anesthetized using isoflurane and quickly decapitated. Coronal slices $(300$ $\mu \mathrm{m})$ containing the LS were prepared using a vibratome (Campden Instruments) and collected in an ice-cold slicing solution (in mM: 10
$\mathrm{NaCl}, 1.2 \mathrm{KCl}, 26 \mathrm{NaHCO}_{3}, 15$ glucose, $1.2 \mathrm{KH}_{2} \mathrm{PO}_{4}, 1 \mathrm{CaCl}_{2}, 2 \mathrm{MgCl}_{2}$, 195 sucrose; osmolality adjusted to $300 \mathrm{mOsmol} / \mathrm{L} ; \mathrm{pH}=7.4,95 \% \mathrm{O}_{2}$ and $5 \% \mathrm{CO}_{2}$ ). Slices were allowed to recover for 1 hour at $37^{\circ} \mathrm{C}$ in artificial cerebrospinal fluid (in mM: $110 \mathrm{NaCl}, 3.6 \mathrm{KCl}, 26 \mathrm{NaHCO}_{3}, 10$ 
glucose, $1.2 \mathrm{KH}_{2} \mathrm{PO}_{4}, 2 \mathrm{CaCl}_{2}, 2 \mathrm{MgCl}_{2}, 0.2$ ascorbic acid, 0.2 thiourea, osmolality adjusted to $300 \mathrm{mOsmol} / \mathrm{L} ; \mathrm{pH}=7.4,95 \% \mathrm{O}_{2}$ and $5 \% \mathrm{CO}_{2}$ ) until placed in the recording chamber perfused at $1 \mathrm{~mL} / \mathrm{min}$ with heated ACSF. Recordings were performed using an Axopatch 200B amplifier (Axon Instruments). $\mathrm{An} \mathrm{Ag} / \mathrm{AgCl}$ electrode placed in the recording chamber was used as reference. A similar electrode was inserted in a borosilicated glass (GC150F-10, Harvard Apparatus) electrode (4-6 Ohm resistance when immersed in the bath) containing intracellular medium (in mM: $9 \mathrm{KCl}, 130 \mathrm{KMeSO}_{3}, 8 \mathrm{NaCl}, 1 \mathrm{MgCl}_{2}, 0.1 \mathrm{EGTA} / \mathrm{Na}$, 10 HEPES/NaOH, 2 pyruvate, 2 malate, $0.5 \mathrm{NaH}_{2} \mathrm{PO}_{4}, 0.5$ cAMP, 2 ATP-Mg, 0.5 GTP-Tris, 14 phosphocreatine, 0.1 leupeptin) and connected to the preamplifier. Neurons visualized using infrared Normarsky contrast microscopy and recorded from the LS were chosen randomly. A bath application of $\operatorname{AVP}(1 \mu \mathrm{M})$ for 2 minutes with or without GABAzine $(1 \mu \mathrm{M})$ or tetrodotoxin (TTX, $0.3 \mu \mathrm{M})$ was performed. Action potentials were recorded in whole-cell or cell-attached configurations for at least 5 minutes before any drug application in current or voltage clamp conditions, respectively. $\mathrm{E}_{\mathrm{Cl}}$ was set to $-50 \mathrm{mV}$ in whole cell recordings. Interspike intervals (ISI) were calculated over a 3-minute period and classified depending on their duration. Data were normalized to the duration of ISI. Alexa Fluor 594 cadaverine $(50 \mu \mathrm{M}$, Life Technologies, Thermo Fisher Scientific) was added to the intracellular medium of the patch pipette and whole-cell recordings performed as usual to determine cell morphology a posteriori.

Immunohistochemistry. Mice were anesthetized with pentobarbital $(50 \mathrm{mg} / \mathrm{kg})$ and perfused at a rate of $3 \mathrm{~mL} / \mathrm{min}$ through the ascending aorta with $30 \mathrm{~mL}$ of ice-cold $0.9 \% \mathrm{NaCl}$ and $4 \%$ ice-cold paraformaldehyde. Brains were sectioned with a vibratome and free-floating coronal sections rinsed in PBS were blocked in 3\% normal donkey serum, PBS, 0.1\% Triton X-100 for 2 hours at $25^{\circ} \mathrm{C}$. Nonlabeled Fab anti-mouse IgG (1:500; Abliance, BI 3013C) was used to block nonspecific sites when mouse antibodies were chosen. Primary antibody dilutions for NG 1:1000, GAD67 1:500, NeuN 1:500 (Merck), c-Fos 1:1000, SST 1:50, Neurotensin 1:250 (Abcam), Calbindin 1:5000, Calretinin 1:1000 (Swant, Inc.), Neurophysin II 1:500 (PS41 from $\mathrm{H}$. Gainer, NIH, Bethesda, Maryland, USA) were incubated overnight. Alexa Fluor-conjugated secondary antibodies (1:2000; Thermo Fisher Scientific) were incubated for 2 hours at $25^{\circ} \mathrm{C}$. Images were acquired with a confocal microscope LSM780 (Carl Zeiss) and 10× or 20× dry objectives or $40 \times$ oil immersion objective for coexpression studies or with an epifluorescence microscope (Imager.Z1, Axiocam, Carl Zeiss). Excitation and acquisition parameters were unchanged during the acquisition of all images. Information about each antibody and other reagents are detailed in Supplemental Table 2.

Image analyses. Images spanning the anterior, central, and posterior septum from the same mouse served to count the number of c-Fos ${ }^{+}$ cells normalized to the surface of the region of interest marked with Image ( $\mathrm{NIH}$ ). The c-Fos ${ }^{+}$cells were further averaged between groups of mice for comparison. Positions of cannulas and fiber optics were determined under the microscope in at least 3 sections of septum in each postmortem brain. More than 67,500 c-Fos ${ }^{+}$cells, 5978 AVP neurons, 2947 SST neurons, and 4491 d[Lys(Alexa Fluor 647) ${ }^{8}$ ]VP-labeled cells were counted in all groups to determine proportions of various cell types colabeled with c-Fos using ImageJ. Semiguided tracing of OXT and AVP fibers into the septum was performed with the NeuronJ plugin.

Synthesis of $d\left[\right.$ Lys(Alexa Fluor 647) $\left.{ }^{8}\right] \mathrm{VP}$. A mixture of $1 \mathrm{mg}$ [Desamino-Cys ${ }^{1}$, Lys $\left.^{8}\right]$ Vasopressin (Bachem) in $100 \mu \mathrm{L}$ of anhydrous dimethylformamide and $2.5 \mu \mathrm{L}$ of $\mathrm{N}, \mathrm{N}$-diisopropylethylamine and $1 \mathrm{mg}$ Alexa Fluor 647 carboxylic acid succinimidyl ester (Thermo Fisher Scientific) was stirred for 5 hours at room temperature in the dark, acidified with $6 \mu \mathrm{L}$ trifluoroacetic acid (TFA) and diluted in 10 $\mathrm{mL}$ water. The lyophilized material was diluted with $3 \mathrm{~mL}$ of $0.05 \%$ TFA in water, purified by semipreparative HPLC on a Hitachi D-7000 HPLC system (detection at $214 \mathrm{~nm}$ ), using a C18 column (Vydac 218TP510 C18, Grace Vydac), solvent A 0.05\% TFA in water, solvent B $0.05 \%$ TFA in acetonitrile, gradient $10 \%$ to $70 \%$ solvent B in solvent A over 30 minutes, flow rate $5.0 \mathrm{~mL} / \mathrm{min}$. Yield of lyophilized d[Lys(Alexa Fluor 647) ${ }^{8}$ ]VP 1.5 mg ( 87\%), RP-HPLC: tR = 15.32 minutes, purity $100 \%$.

Quantitative binding of $\left.d[\text { Lys(Alexa Fluor 647) })^{8}\right] V P$. HEK293T cells were grown in DMEM supplemented with $10 \%$ bovine serum and transfected with recombinant murine AVPR1b and OXTR (Origene: AVPR1b: MR217375; OXTR: MR225679) using lipofectamine (Thermo Fisher Scientific). Membranes were extracted from transfected cells and from mouse liver and kidney medulla rich in AVPR1a and AVPR2, respectively. The affinity of d[Lys(Alexa Fluor 647) ${ }^{8}$ ]VP was determined by competitive binding assays with $1 \mathrm{nM}$ of $\left[{ }^{3} \mathrm{H}\right] \mathrm{AVP}$ (PerkinElmer) in triplicates in at least $n=3$ independent experiments. $\mathrm{K}_{\mathrm{i}}$ values for d[Lys(Alexa Fluor 647) ${ }^{8}$ ]VP were calculated from dose response curves.

Cytological binding specificity of $d\left[\right.$ Lys(Alexa Fluor 647) $\left.{ }^{8}\right] V P$. Acute brain slices were prepared and maintained as described earlier. Slices were maintained at $12^{\circ} \mathrm{C}$ in ACSF and perfused at $1 \mathrm{~mL} /$ min with $150 \mathrm{nM}$ d[Lys(Alexa Fluor 647$)^{8}$ ] VP for 1 hour at $12^{\circ} \mathrm{C}$, followed by 3 rinses with ACSF at $12^{\circ} \mathrm{C}$ and finally fixation with $4 \%$ PFA. Nonspecific binding monitored with Manning compound $(5 \mu \mathrm{M})$ served as reference control for excitation and acquisition parameters of images using epifluorescence microscopy. Images illustrated are representative of at least 3 distinct experiments for which at least 100 cells were analyzed.

AVPR1a autoradiography. Mouse brains were frozen in isopentane vapors cooled with dry ice. Next, $20 \mu \mathrm{m}$ thick coronal slices obtained with a cryostat were placed on Superfrost Plus slides (Thermo Fisher Scientific) stored at $-80^{\circ} \mathrm{C}$. Sections were blocked with $50 \mathrm{mM}$ Tris $\mathrm{HCl}, 1 \mathrm{mM}$ EDTA, and $1 \mathrm{mg} / \mathrm{mL}$ BSA before addition of $50 \mathrm{pM}$ LVA-[I25] (PerkinElmer) for 2 hours at room temperature under a wet atmosphere with (nonspecific) or without (total) $1 \mu \mathrm{M}$ AVP. Sections were rinsed and autoradiography revealed after 24 hours' exposure on a photographic film (BioMax MR film, Carestream Health). The optical densities in the regions of interest were measured with ImageJ. Each measurement was subtracted by tissue background, and binding densities were calculated by taking the mean of 6 measurements from bilateral structures. Displacement of LVA-[1'125] labeling $(100 \%$ $\pm 5.9 \%$, mean \pm SEM) up to $63 \% \pm 0.2 \%$ by $1 \mu \mathrm{M}$ Manning compound $(P<0.0001), 2 \% \pm 2.13 \%$ by $100 \mathrm{nM}$ TGOT $(P=0.8)$. Effect of displacement was analyzed with 1 -way ANOVA $F_{2,15}=103, P<0.0001$, post hoc Dunnett test.

Statistics. Data are shown as mean \pm SEM and are statistics presented in Supplemental Table 3. Estimates of sample size were calculated by power analysis based on preliminary data. Sample size was chosen to ensure $80 \%$ power and detect the prespecified effect size. The $\alpha$ was set at 0.05 and multiple comparisons controlled for the type I error. Representation of $n$ for each data set is indicated in figure legends. All data collected in animals were from littermate controls 
and averaged per experimental groups. Data were considered nonparametric when sample size was moderate. Data were tested for normality and compared with a theoretical value with Wilcoxon signed rank test (Prism 8.0 Software, GraphPad). We used factorial ANOVA to compare multiple groups (behavior, genotype, optogenetics, and pharmacology) followed by post hoc pairwise comparisons with Sidak test. The randomization process for pharmacology experiments in vivo and in vitro was that animals received the different compounds in a random order. The following were the inclusion or exclusion criteria: (a) postmortem validation of positions of cannulas, (b) optic fibers, and (c) virus-mediated recombination. An independent experimenter blind to the conditions performed these analyses. The following were the preestablished criteria for excluding data: (a) misposition of cannulas or fiber optics, (b) poor recombination of virus-guided tools, and (c) instability of EEG signal. The following were the preestablished criteria for stopping data collection: (a) mice reaching ethical endpoint limits and (b) unexpected detachment of cranial implants.

Study approval. All experiments were carried out in accordance with the Directive by the Council of the European Communities (86/609/EEC). The study was approved by and followed guidelines from the French ministry of research and ethics committee for the care and use of laboratory animals (approved protocol APAFIS 5133,
$8940,11468)$. All efforts were made to minimize animal suffering and to reduce the number of mice utilized in each series of experiments.

\section{Author contributions}

$\mathrm{AMB}, \mathrm{MGD}$, and FJ were responsible for conceptualization and methodology; AMB, YD, MM, OA, MGD, GG, and FJ conducted the investigation and formal analyses; FJ, MGD, and AMB wrote the manuscript; GG and FM provided critical feedback; FJ, MGD, and FM performed administration and supervision and acquired funding. All authors reviewed and approved the final manuscript.

\section{Acknowledgments}

We thank ANR (MGD, FM); Fondation Lejeune (MGD); Fondation pour la recherche médicale (FJ, AMB); Montpellier University (AMB); N. Marchi and B. Boussadia (IGF, Montpellier) for helping with the EEG recordings; M. Arango-Lievano for technical strategies; and M. Tauber (CHU Toulouse) for discussions on PWS.

Address correspondence to: Freddy Jeanneteau, Institut de Génomique Fonctionnelle, 141 rue de la Cardonille, 34094 Montpellier, France. Phone: 33.4.3435.9293; Email: freddy.jeanneteau@igf.cnrs.fr.
1. American Psychiatric Association. Diagnostic and Statistical Manual of Mental Disorders. 5th ed. Washington, DC: American Psychiatric Association; 2013

2. Masi A, et al. An overview of autism spectrum disorder, heterogeneity and treatment options. Neurosci Bull. 2017;33(2):183-193.

3. Chaste P, Leboyer M. Autism risk factors: genes, environment, and gene-environment interactions. Dialogues Clin Neurosci. 2012;14(3):281-292.

4. Satterstrom FK, et al. Large-scale exome sequencing study implicates both developmental and functional changes in the neurobiology of autism. Cell. 2020;180(3):568-584.e23.

5. Salles J, et al. The RDoC approach for translational psychiatry: could a genetic disorder with psychiatric symptoms help fill the matrix? the example of Prader-Willi syndrome. Transl Psychiatry. 2020;10(1):274.

6. Dykens EM, et al. Prader-Willi syndrome and autism spectrum disorders: an evolving story. J Neurodev Disord. 2011;3(3):225-237.

7. Schaaf CP, et al. Truncating mutations of MAGEL2 cause Prader-Willi phenotypes and autism. Nat Genet. 2013;45(11):1405-1408.

8. Fountain MD, Schaaf CP. Prader-Willi syndrome and Schaaf-Yang syndrome: neurodevelopmental diseases intersecting at the MAGEL2 gene. Diseases. 2016;4(1):2.

9. Kamaludin AA, et al. Muscle dysfunction caused by loss of Magel 2 in a mouse model of PraderWilli and Schaaf-Yang syndromes. Hum Mol Genet. 2016;25(17):3798-3809.

10. Maillard J, et al. Loss of Magel2 impairs the development of hypothalamic anorexigenic circuits. Hum Mol Genet. 2016;25(15):3208-3215.

11. Patak J, et al. MAGEL2-related disorders: a study and case series. Clin Genet. 2019;96(6):493-505.

12. Angulo MA, et al. Prader-Willi syndrome: a review of clinical, genetic, and endocrine find- ings. J Endocrinol Invest. 2015;38(12):1249-1263.

13. Fountain MD, et al. Magel2 knockout mice manifest altered social phenotypes and a deficit in preference for social novelty. Genes Brain Behav. 2017;16(6):592-600.

14. Meziane $\mathrm{H}$, et al. An early postnatal oxytocin treatment prevents social and learning deficits in adult mice deficient for Magel2, a gene involved in Prader-Willi syndrome and autism. Biol Psychiatry. 2015;78(2):85-94.

15. Schaller F, et al. A single postnatal injection of oxytocin rescues the lethal feeding behaviour in mouse newborns deficient for the imprinted Magel2 gene. Hum Mol Genet. 2010;19(24):4895-4905.

16. Tauber M, et al. The use of oxytocin to improve feeding and social skills in infants with Prader-Willi syndrome. Pediatrics. 2017;139(2):e20162976.

17. Bernaerts S, et al. Behavioral effects of multiple-dose oxytocin treatment in autism: a randomized, placebo-controlled trial with long-term follow-up. Mol Autism. 2020;11(1):6.

18. Wagner S, Harony-Nicolas H. Oxytocin and animal models for autism spectrum disorder. Curr Top Behav Neurosci. 2018;35:213-237.

19. Penagarikano O, et al. Exogenous and evoked oxytocin restores social behavior in the Cntnap2 mouse model of autism. Sci Transl Med. 2015;7(271):271ra278.

20. Peñagarikano O. Oxytocin in animal models of autism spectrum disorder. Dev Neurobiol. 2017;77(2):202-213

21. Song Z, Albers HE. Cross-talk among oxytocin and arginine-vasopressin receptors: relevance for basic and clinical studies of the brain and periphery. Front Neuroendocrinol. 2018;51:14-24.

22. Parker KJ, et al. A randomized placebo-controlled pilot trial shows that intranasal vasopressin improves social deficits in children with autism. Sci Transl Med. 2019;11(491):eaau7356.
23. Bolognani $\mathrm{F}$, et al. A phase 2 clinical trial of a vasopressin V1a receptor antagonist shows improved adaptive behaviors in men with autism spectrum disorder. Sci Transl Med. 2019;11(491):eaat7838.

24. Tendler A, Wagner S. Different types of theta rhythmicity are induced by social and fearful stimuli in a network associated with social memory. Elife. 2015;4:e03614.

25. Raam T, et al. Hippocampal oxytocin receptors are necessary for discrimination of social stimuli. Nat Commun. 2017;8(1):2001.

26. Smith AS, et al. Targeted activation of the hippocampal CA2 area strongly enhances social memory. Molecular Psychiatry. 2016;21(8):1137-1144.

27. Trainor BC, Hofmann HA. Somatostatin regulates aggressive behavior in an African cichlid fish. Endocrinology. 2006;147(11):5119-5125.

28. $\mathrm{Xu} \mathrm{H}$, et al. A disinhibitory microcircuit mediates conditioned social fear in the prefrontal cortex. Neuron. 2019;102(3):668-682.e5.

29. Scheggia D, et al. Somatostatin interneurons in the prefrontal cortex control affective state discrimination in mice. Nat Neurosci. 2020;23(1):47-60.

30. Manning M, et al. Oxytocin and vasopressin agonists and antagonists as research tools and potential therapeutics. J Neuroendocrinol. 2012;24(4):609-628.

31. Chen $\mathrm{H}$, et al. Loss of MAGEL2 in Prader-Will syndrome leads to decreased secretory granule and neuropeptide production. JCI Insight. 2020;5(17):e138576.

32. Lukas M, et al. Early life stress impairs social recognition due to a blunted response of vasopressin release within the septum of adult male rats. Psychoneuroendocrinology. 2011;36(6):843-853.

33. Chini $\mathrm{B}$, et al. The action radius of oxytocin release in the mammalian CNS: from single vesicles to behavior. Trends Pharmacol Sci. 2017;38(11):982-991. 
34. Sala M, et al. Pharmacologic rescue of impaired cognitive flexibility, social deficits, increased aggression, and seizure susceptibility in oxytocin receptor null mice: a neurobehavioral model of autism. Biol Psychiatry. 2011;69(9):875-882.

35. Sheehan TP, et al. Regulation of affect by the lateral septum: implications for neuropsychiatry. Brain Res Rev. 2004;46(1):71-117.

36. Besnard A, et al. Dorsolateral septum somatostatin interneurons gate mobility to calibrate context-specific behavioral fear responses. Nat Neurosci. 2019;22(3):436-446.

37. Dong HW, Swanson LW. Projections from bed nuclei of the stria terminalis, anteromedial area: cerebral hemisphere integration of neuroendocrine, autonomic, and behavioral aspects of energy balance. JComp Neurol. 2006;494(1):142-178.

38. Trainor B, et al. Individual differences in estrogen receptor alpha in select brain nuclei are associated with individual differences in aggression. Horm Behav. 2006;50(2):338-345.

39. Clauss JA, et al. Social anxiety is associated with BNST response to unpredictability. Depress Anxiety. 2019;36(8):666-675.
40. Rigney N, et al. Sexually dimorphic vasopressin cells modulate social investigation and communication in sex-specific ways. eNeuro. 2019;6(1):ENEURO.0415-0418.2019.

41. Lebow M, Chen A. Overshadowed by the amygdala: the bed nucleus of the stria terminalis emerges as key to psychiatric disorders. Mol Psychiatry. 2016;21(4):450-463.

42. Pallett PM, et al. Face and object discrimination in autism, and relationship to IQ and age. JAutism Dev Disord. 2014;44(5):1039-1054.

43. Ito-Ishida A, et al. Loss of MeCP2 in parvalbumin- and somatostatin-expressing neurons in mice leads to distinct Rett syndrome-like phenotypes. Neuron. 2015;88(4):651-658.

44. Vogt D, et al. The parvalbumin/somatostatin ratio is increased in Pten mutant mice and by human PTEN ASD alleles. Cell Rep. 2015;11(6):944-956.

45. Chen G, et al. Distinct inhibitory circuits orchestrate cortical $\beta$ and $\gamma$ band oscillations. Neuron. 2017;96(6):1403-1418.e6.

46. Urban IJ. Effects of vasopressin and related peptides on neurons of the rat lateral septum and ventral hippocampus. Prog Brain Res.
1998;119:285-310.

47. Rilling JK, et al. Arginine vasopressin effects on subjective judgments and neural responses to same and other-sex faces in men and women. Front Endocrinol (Lausanne). 2017;8:200.

48. Festante F, et al. Intranasal oxytocin enhances EEG mu rhythm desynchronization during execution and observation of social action: an exploratory study. Psychoneuroendocrinology. 2020;111:04467.

49. Scheyltjens I, Arckens L. The current status of somatostatin-interneurons in inhibitory control of brain function and plasticity. Neural Plast. 2016;2016:8723623.

50 . McCarthy J, et al. Schaaf-Yang syndrome overview: report of 78 individuals. Am JMed Genet $A$. 2018;176(12):2564-2574.

51. Engelmann M, et al. Social discrimination procedure: an alternative method to investigate juvenile recognition abilities in rats. Physiol Behav. 1995;58(2):315-321.

52. Arango-Lievano M, et al. Topographic reorganization of cerebrovascular mural cells under seizure conditions. Cell Rep. 2018;23(4):1045-1059. 